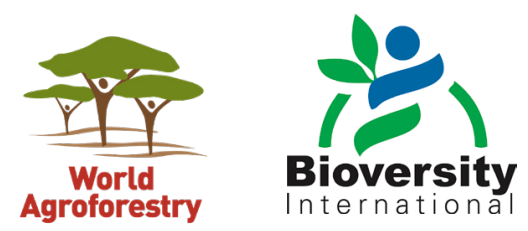

Fuentes semilleras y especies forestales de los bosques secos tropicales del norte del Perú: Estado actual y prioridades futuras

Jéssica Cerrón, Rachel Atkinson, Evert Thomas, Jonathan P. Cornelius

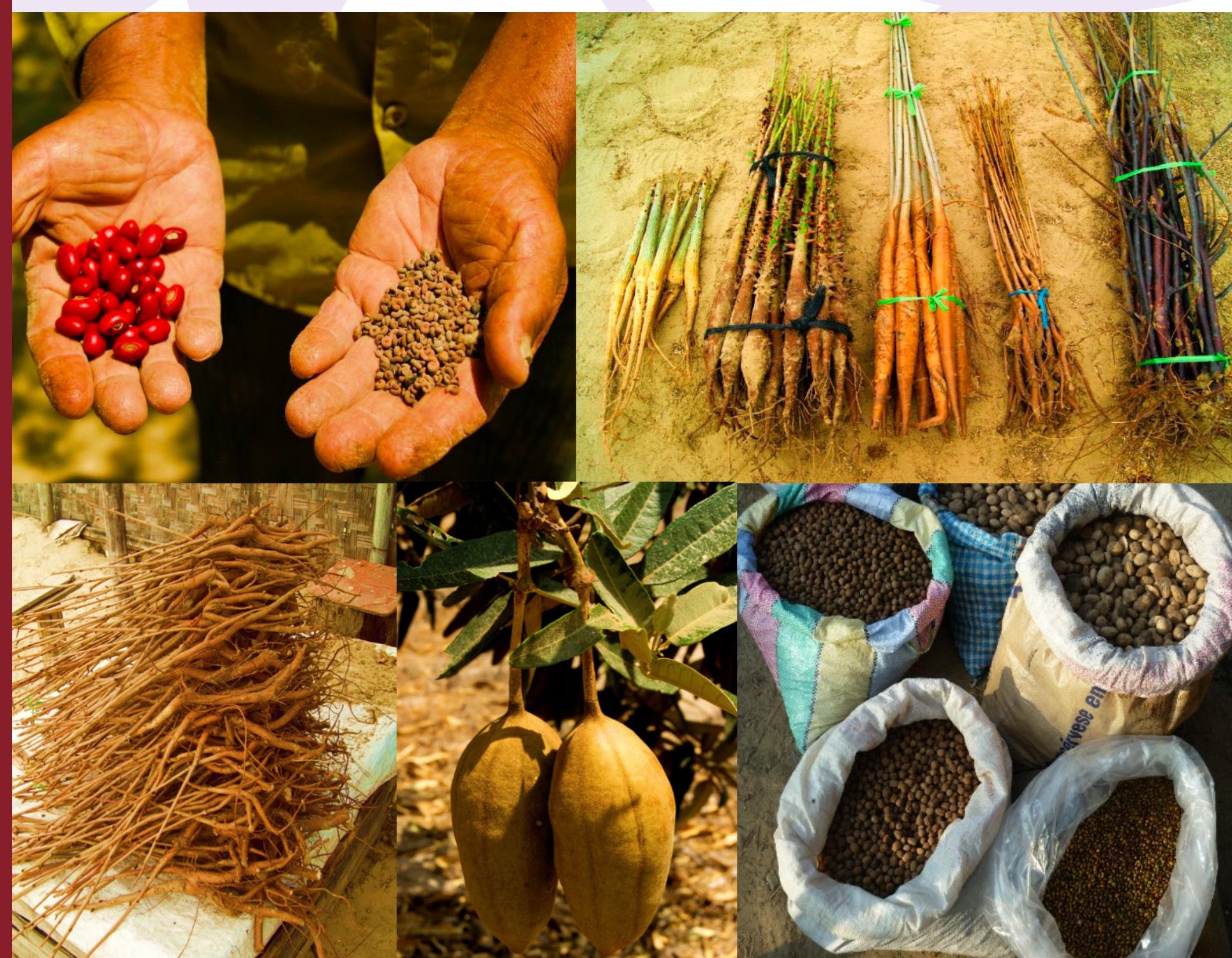


Fuentes semilleras y especies forestales de los bosques secos tropicales del norte del Perú: Estado actual y prioridades futuras

Jéssica Cerrón, Rachel Atkinson, Evert Thomas, Jonathan P. Cornelius 


\section{Cita correcta:}

Cerrón J., Atkinson R., Thomas E., Cornelius J.P. 2019. Fuentes semilleras y especies forestales de los bosques secos tropicales del norte del Perú: estado actual y prioridades futuras. Documento de Trabajo número 301. Centro Internacional de Investigación Agroforestal, Lima, Perú.

DOI: http://dx.doi.org/10.5716/WP19057.PDF

Los títulos de la Serie de Documentos de Trabajo tienen por objeto difundir los resultados provisionales de la investigación y las prácticas agroforestales y estimular la retroalimentación de la comunidad científica. Otras series de publicaciones del Centro Internacional de Investigacición Agroforestal incluyen Manuales Técnicos, Documentos Ocasionales y la Serie 'Árboles para el Cambio'

Publicado por el Centro Internacional de Investigación Agroforestal

United Nations Avenue

PO Box 30677, GPO 00100

Nairobi, Kenya

Tel: $+254(0) 207224000$, via USA +1 6508336645

Fax: +254(0)20 7224001, via USA +1 6508336646

Email: worldagroforestry@cgiar.org

Website: www.worldagroforestry.org

(C) Centro Internacional de Investigación Agroforestal 2019

Documento de Trabajo No 301

Fotos: Las opiniones expresadas en esta publicación son las de los autores y no necesariamente las del Centro Internacional de Investigación Agroforestal. Esta publicación pueden ser citados o reproducidos sin costo alguno, siempre que se cite la fuente. Todas las imágenes son propiedad exclusiva de su fuente y no pueden ser utilizadas para ningún propósito sin el permiso escrito de la fuente. 


\section{Acerca de los autores}

Jéssica M. Cerrón-Macha

Consultora Forestal de Centro Internacional de Investigación Agroforestal (ICRAF), Lima, Perú (jessicamcerronm@gmail.com), e Ingeniera Forestal por la Universidad Nacional Agraria La Molina, Lima, Perú. Actualmente trabajando en el impacto de las diferentes prácticas de restauración sobre los servicios ecosistémicos y los modos de vida locales en Perú.

\section{Rachel Atkinson}

Científica Asociada de Bioversity International, Apartado 1558, Lima 12, Perú (R.Atkinson@cgiar.org) y Especialista en Restauración de Ecosistemas. Británica con 20 años trabajando en conservación y restauración de bosques degradados en América Latina.

\section{Evert Thomas}

Científico de Bioversity International, Apartado 1558, Lima 12, Perú (E.Thomas@cgiar.org) y Especialista en Conservación y uso de los recursos genéticos forestales en América Latina. Sus principales intereses de investigación actualmente son la restauración de Bosques Tropicales Estacionalmente Secos del Perú, mejora de la comprensión de la diversidad nativa de los recursos genéticos del cacao (Theobroma cacao) y la nuez del Brasil (Bertholletia excelsa) en el Perú.

Jonathan Philip Cornelius Investigador y Comunicador Sénior de Centro Internacional de Investigación Agroforestal, CIPICRAF, Apartado 1558, Lima 12, Perú (j.cornelius@cgiar.org), y Profesor Asociado Adjunto, Universidad James Cook, Cairns, Queensland, Australia. 


\section{Resumen}

El presente documento tiene el objetivo de proporcionar un análisis de las fuentes semilleras existentes en el bosque seco tropical del norte del Perú (bosques secos de Tumbes-Piura y bosques secos del Marañon), además de proveer información preliminar del estado de amenaza y/o conservación de especies forestales de interés y aquellas utilizadas en experiencias de reforestación, restauración entre otros para la planificación e implementación de futuras actividades en el marco del Proyecto "Fuentes semilleras agroforestales para la restauración y conservación genética (FuenteS)" en estas ecorregiones.

En ese contexto, se realizó la recopilación de información de fuentes semilleras existentes a partir de un cuestionario estructurado y completado con información procedente de fuentes primarias y secundarias, y en el caso de especies forestales en estado de amenaza, se determinó una lista de interés en función a la indagación en categorizaciones oficiales y consulta a actores claves de cada ecorregión. Entre los principales resultados que se encontraron fue la identificación de 45 fuentes semilleras de 16 especies solo en los bosques secos de Tumbes-Piura y ninguna en los bosques secos del Marañon, las fuentes existentes están bajo propiedad de las comunidades campesinas principalmente, siendo la mayoría de éstas de las especies Prosopis pallida (47\%) y Bursera graveolens (11\%); sin embargo, existen 27 fuentes semilleras potenciales en las áreas de conservación. De otro lado, en el caso de especies forestales en estado de conservación o amenaza, se identificaron preliminarmente 12 especies bajo diferentes categorías oficiales y 23 especies determinadas por expertos locales para los Bosques Secos de Tumbes-Piura; y en el caso de los Bosques Secos del Marañon, siete especies en categorias oficiales y 21 de interés potencial.

\section{Palabras clave}

Bosques secos, bosques secos de Tumbes-Piura, bosques secos del Marañon, fuentes semilleras, especies forestales en conservación, especies forestales amenazadas 


\section{Agradecimientos}

Queremos expresar nuestro profundo agradecimiento a todas aquellas personas que contribuyeron en la elaboración del presente estudio. Destacamos la colaboración y la respuesta efectiva de los actores de las diversas instituciones que brindaron información y documentos de fuentes semilleras y de especies forestales en estado de conservación o amenaza.

Asimismo, este documento es producto del proyecto "Fuentes Semilleras Agroforestales para la Restauración y Conservación Genética (FuenteS)" que viendo siendo desarrollado por ICRAF en colaboración con INIA y SERFOR, financiado por el Ministerio de Agricultura y Riego del Peru a través de su Secretaría de Cooperación con el CGIAR (STC-CGIAR) y del proyecto "Hacia una restauración climáticamente inteligente: una caja de herramientas para el Bosque Seco Tropical de Perú 2018-2020" financiado por el Ministerio Federal de Cooperación Económica y Desarrollo, Alemania a través del programa BEAF del GIZ. 


\section{Contenido}

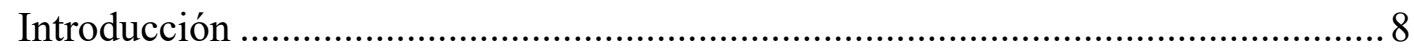

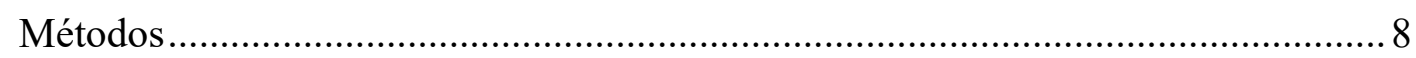

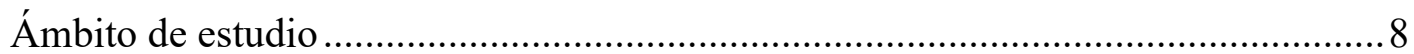

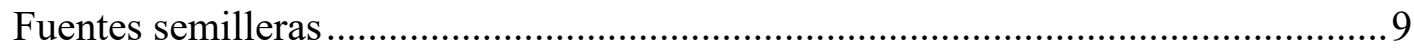

Especies forestales en estado de conservación o amenaza ................................... 10

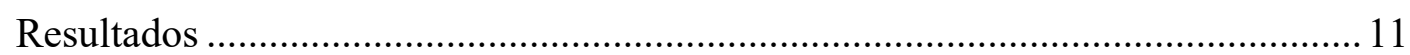

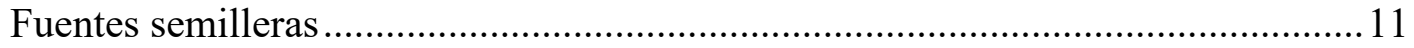

Especies forestales en estado de conservación o amenaza .................................... 16

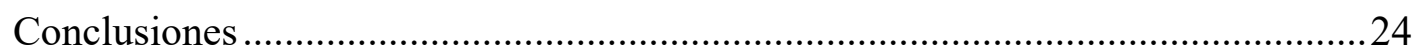

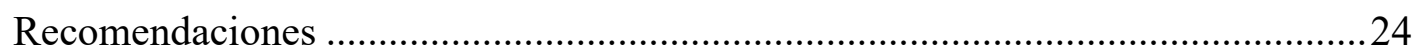

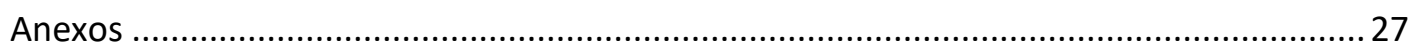

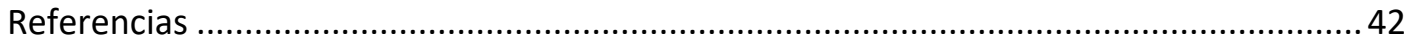

Documentos de Trabajo de ICRAF (Working Paper Series) ................................................ 45 


\section{Acrónimos}

CITES Convención sobre el Comercio Internacional de Especies Amenazadas de Fauna y Flora Silvestre

COSUDE Agencia Suiza para el Desarrollo y la Cooperación

EEA Estación Experimental Agraria de INIA

FOSEFOR Programa Andino de Fomento de Semillas Forestales

INIA Instituto Nacional de Innovación Agraria, Peru

IUCN Unión Internacional para la Conservación de la Naturaleza

MINAGRI Ministerio de Agricultura y Riego, Perú

MINAM Ministerio del Ambiente, Perú

SENASA Servicio Nacional de Sanidad Agraria, Perú

SERFOR Servicio Nacional Forestal y de Fauna Silvestre, Perú 


\section{Introducción}

Los bosques secos tropicales del norte del Perú presentan una extensión total de 3,4 M ha, de las cuales el $63 \%$ se encuentra en degradación (MINAM 2011) como consecuencia de la deforestación, la tala selectiva, la agricultura y la ganadería caprina y bovina (Angulo 2009). En dicho contexto, durante los últimos años se han realizando diversas iniciativas con el propósito de contrarrestar el proceso de degradación y la pérdida de áreas boscosas, tales como: reforestación, manejo de regeneración natural, conservación, agroforestería entre otras. Sin embargo, el material de siembra utilizado en estas intervenciones mayormente no procede de fuentes semilleras identificadas, sino como resultado de una colecta improvisada de semillas, en la mayoría de casos para cubrir la demanda de solo algunas especies de interés comercial y sujeta a los conocimientos técnicos silviculturales de éstas. Asimismo, el uso desmedido de algunas especies sin reposisión de éstas ha conducido a que varias de ellas se encuentren actualmente en diferentes categorías de conservación o amenaza.

En dicho contexto, el presente documento tiene los siguientes objetivos:

- Proporcionar un análisis de las fuentes semilleras existentes en el bosque seco tropical del norte del Perú (bosques secos de Tumbes-Piura y bosques secos del Marañon).

- Proveer información del estado de amenaza y/o conservación de especies forestales de interés y aquellas utilizadas en experiencias de reforestación y restauración.

- Facilitar la reforestación y restauración a través del fortalecimiento de capacidades, investigación y transferencia de tecnología a los actores claves.

\section{Métodos}

\section{Ámbito de estudio}

Se realizó la recopilación de información de las fuentes semilleras existentes y de las especies forestales en estado de conservación o amenaza de las ecorregiones bosques secos de Tumbes-Piura, en los departamentos de Lambayeque, Tumbes y Piura y de los bosques secos del Marañon en Cajamarca y Amazonas.

\section{Bosques secos de Tumbes-Piura}

Se extienden a lo largo de la costa norte del Perú, desde el nivel del mar hasta 1500 m s.n.m. (Linares-Palomino 2004) en los departamentos de Piura, Tumbes, Lambayeque y pequeñas porciones de La Libertad y Cajamarca, con una extensión total de 3 235,012 ha (MINAM 2011). Especies arbóreas como el algarrobo (Prosopis pallida), el sapote (Colicodendron scabridum), el faique (Vachellia macracantha), y el overo (Cordia lutea) dominan la zona de llanura. En las partes altas, se presenta una mayor diversidad de árboles, entre los cuales destacan el palo 
santo (Bursera graveolens), el hualtaco (Loxopterygium huasango) y el pasallo (Eriotheca ruizii), entre muchos otros (Angulo 2009).

\section{Bosques secos del Marañon}

Se extienden a lo largo de la cuenca del Río Marañon, el principal afluente del Amazonas, entre los 400 y 2200 m s.n.m. (Marcelo-Peña et al. 2015) en los departamentos de Ancash, Huánuco, La Libertad, Cajamarca, Amazonas y Piura con una extensión total de 372915 ha (MINAM 2011). Presentan especies arbóreas tales como el acerillo (Aspidosperma polyneuron), el iguaguana (Cordia iguaguana), y arbustivas como Calliandra mollissima, Coursetia maraniona, Jatropha humboldtiana (el huanarpo), entre otras especies. Se considera que el $33 \%$ de las especies de plantas de esta ecorregión son endémicas y presenta numerosas especies con alto potencial para reforestación en zonas áridas y como estabilizadoras de suelos (Marcelo-Peña et al. 2015).

\section{Fuentes semilleras}

La información de las fuentes semilleras se recopiló en dos formularios, uno para aquellas fuentes ubicadas en áreas de conservación (nacional, regional y privada) y otra para aquellas que se ubican en otras zonas. Los dos formularios abarcan las principales características de éstas (Anexo 1 y Anexo 2) que son: (1) datos de la persona responsable, (2) características del área y (3) características de la fuente de semillas.

La información recopilada de las fuentes existentes fue sistematizada en funcíon a las siguientes variables:

- Tipo de propietario: Se utilizaron las categorías "Entidad Estatal" (instituciones del gobierno nacional, regional y local), "Asociación o Cooperativa" (asociaciones o cooperativas locales registradas), "Predio Privado" (actor local individual o familiar) y "Comunidad Campesina" (comunidades locales).

- Período desde su establecimiento: Determinado en función a la diferencia entre el año de mapeo (2018) y la fecha de establecimiento de los árboles semilleros.

- Superficie: Área (ha) donde se encuentran distribuidos los árboles semilleros.

- Densidad: Número total de individuos inventariados de la especie por ha.

- Tamaño poblacional: Calculado a partir de la información disponible de superficie (ha) y densidad (árboles/ha) de cada fuente de semillas.

- Número de árboles seleccionados: Cantidad de árboles seleccionados como semilleros del total existente (información facilitada por propietarios).

- Tipo de fuente de semillas: Determinado en función a la información de densidad por ha disponible para cada fuente, considerando la clasificación de MINAGRI (2006) y Cuellar et al. (2016) (Anexo 3). 
- Tipo de material reproductivo extraido: Se utilizaron dos categorías: (1) semillas y (2) estacas.

Cabe resaltar que en esta recopilación, las fuentes semilleras existentes son individuos maduros de especies forestales de hábito arbóreo y/o arbustivo identificados a partir de características fenotípicas. De otro lado, se determinó fuentes semilleras potenciales ${ }^{1}$ en el contexto de áreas de conservación nacional, regional y privadas.

\section{Especies forestales en estado de conservación o amenaza}

Se determinó una lista de especies forestales para los dos tipos de bosque seco: bosques secos de Tumbes-Piura y bosques secos del Marañon.

\section{Bosques secos de Tumbes-Piura}

La lista de especies leñosas fue determinada en función a su uso en experiencias de restauración y la lista de fuentes semilleras existentes recopiladas. De las especies citadas se buscó en qué categoría de conservación y/o amenaza se encuentra, para lo cual se utilizó las categorizaciones internacionales según IUCN (2018) y Apéndices CITES (2016) y para la categorización oficial para el Perú se utilizó la publicación preliminar de los Anexo I y II que contiene la Clasificación Oficial de Especies de Flora Silvestre categorizadas como amenazadas (Resolución Ministerial 0505-2016-MINAGRI), la cual se encuentra actualmente siendo evaluada para su aprobación official. Asimismo, se entrevistó a expertos locales (profesionales y comuneros) acerca de qué especies consideran que se encuentran en estado de amenaza, las cuales se clasificaron en función a los principales factores que influyen en su estado actual.

\section{Bosques secos del Marañon}

En esta ecorregión, hay pocas experiencias de restauración y fuentes semilleras. Por lo tanto, la lista de especies leñosas de interés para su conservación fue determinada en función a su estado de amenaza y endemismo, a partir de la revision bibliográfica de algunos estudios de composición florística (Marcelo-Peña 2008, Marcelo-Peña et al. 2010). De la lista resultante de especies, se buscó en qué categoría de conservación y/o amenaza se encuentra, para lo cual se utilizó las categorizaciones internacionales según IUCN (2018) y Apéndices CITES (2016) y para la categorización oficial para el Perú se utilizó la publicación preliminar de los Anexo I y II que contiene la Clasificación Oficial de Especies de Flora Silvestre categorizadas como amenazadas (Resolución Ministerial 0505-2016-MINAGRI) y las categorías citadas por

\footnotetext{
1 Áreas con presencia de especies leñosas maduras de individuos con características fenotípicas deseables para ser considerados semilleros que según actores locales, pero que aún no han sido identificadas para tal propósito.
} 
Marcelo-Peña et al. (2010). Asimismo, en el caso de las especies endémicas se utilizó los estudios de León et al. (2006), Marcelo-Peña et al. (2010) y Marcelo-Peña et al. (2015). De otro lado, al no identificarse actores locales con suficiente conocimiento de las especies en esta ecorregión, no se elaboró una lista citada por expertos locales; sin embargo, a partir de la revisión bibliográfica del estudio de composición florística realizado por Marcelo-Peña et al. (2010) se logró determinar una lista de especies de interés por su uso bajo diversas prácticas agroforestales.

\section{Resultados}

\section{Fuentes semilleras}

\section{Distribución de fuentes semilleras existentes ( $N=45)$}

En la Figura 1 se aprecia la distribución de las 45 fuentes semilleras existentes, observándose que solo se identificaron en la ecorregión bosques secos de Tumbes-Piura, donde predominó Lambayeque con 22 fuentes y, en menor proporción, Piura y Tumbes; asimismo, no se identificó ninguna fuente semillera en la ecorregión bosques secos del Marañon. Por lo tanto la siguiente caracterización está en función a la ecorregión bosques secos de Tumbes-Piura.

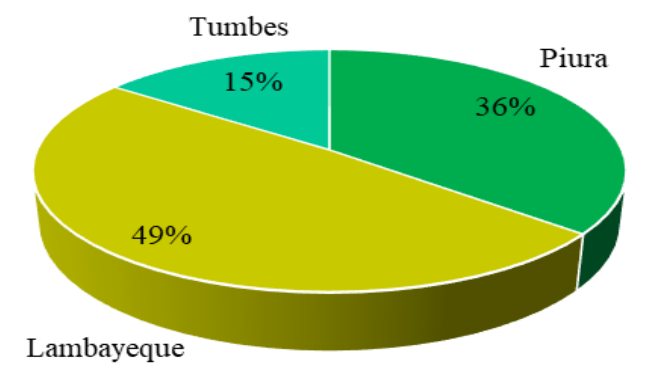

Figura 1. Distribución de fuentes semilleras existentes $(\mathrm{N}=45)$

\section{Tipo de propietario y período desde su establecimiento ( $N=45$ )}

En la Figura 2 se aprecia el predominio de las comunidades campesinas en términos de propiedad con 25 fuentes, donde resaltaron las fuentes semilleras con períodos desde su establecimiento de 3 a 5 años y de 6 a 15 años (12 y 11 respectivamente). El segundo lugar se encuentra representado por los predios privados a nivel local con 12 fuentes, donde predominó el período de 1 a 2 años, ello se debe a que son árboles maduros que fueron seleccionados recientemente como semilleros en el marco de los Planes de Manejo para el Aprovechamiento de los Bosques Secos (principalmente a partir del año 2016). 
Durante el año 2003, el Programa Andino de Fomento

de Semillas Forestales (FOSEFOR), financiado por COSUDE identificó siete fuentes semilleras en Piura, siendo dos de éstas ubicadas en propiedad de la Asociación Agraria Manga Manga de Salitral, las fuentes identificadas fueron de las especies: palo santo (Bursera graveolens) y hualtaco (Loxopterygium huasango). Los árboles semilleros fueron identificados en base a sus características fenotípicas y al uso potencial de las mismas.

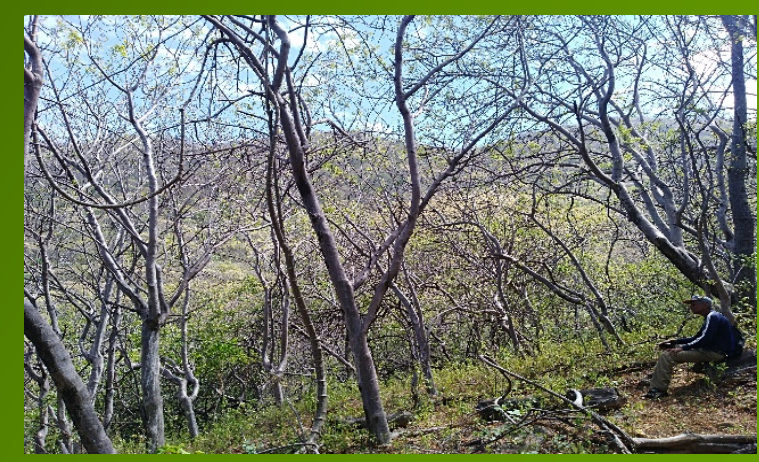

Estado actual de fuente semillera de Bursera graveolens. Fotografía: Jéssica Cerrón
En menor proporción, se presentaron

fuentes semilleras que son administradas por una asociación o cooperativa, presentando dos de éstas períodos de 6 a 15 años, que fueron identificadas anteriormente en el marco de un proyecto que tuvo como fin fortalecer las capacidades locales para la producción de semillas de seis especies forestales de los bosques secos en Piura. Por último, en el caso de las entidades estatales, estas son aquellas que son administradas por INIA-EEA Vista Florida y la Refugio de Vista Silvestre de Laquipampa para las especies algarrobo (Prosopis pallida) y tara (Caesalpinia spinosa) en los dos casos.

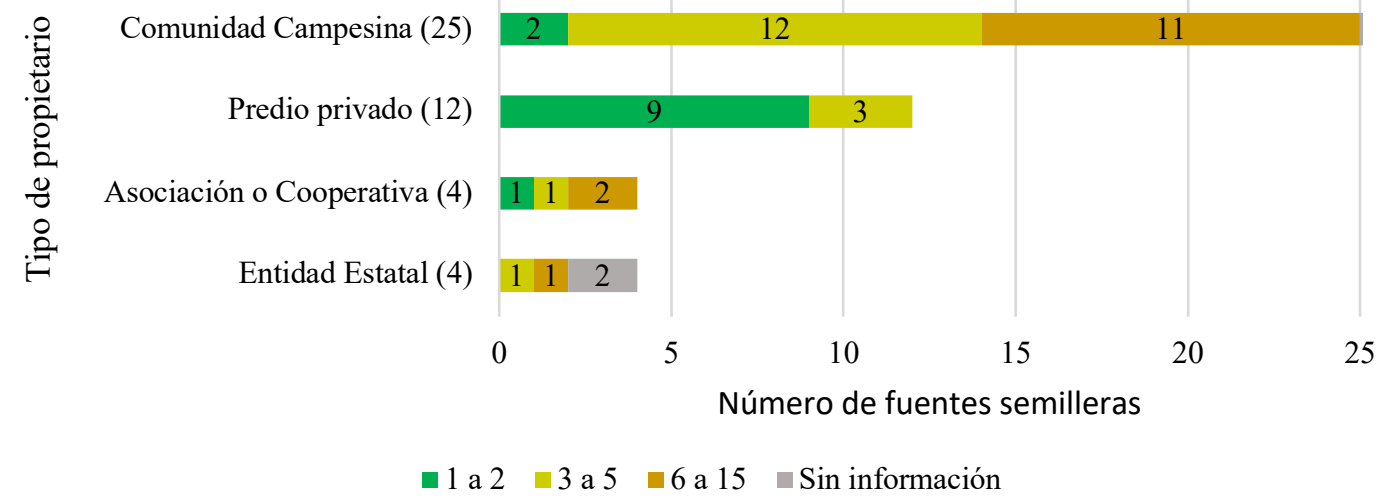

Figura 2. Número de fuentes semilleras por tipo de propietario y período desde su establecimiento $(\mathrm{N}=45)$ 


\section{Especies y período desde su establecimiento $(\mathrm{N}=45)$}

En el caso de las especies, en la Figura 3 se observa la predominancia del algarrobo (Prosopis pallida) con $47 \%$ (21 de 45), donde resaltó el período desde su establecimiento de 1 a 2 años con 11 fuentes (identificados recientemente en el marco de Planes de Manejo principalmente). En segundo lugar, se aprecia al palo santo (Bursera graveolens), que presentó fuentes en los tres períodos sin diferencia significativa. De otro lado se observa que, el rango de 6 a 15 años solo se presentó en algunas especies, tales como: sapote (Colicodendron scabridum), charán

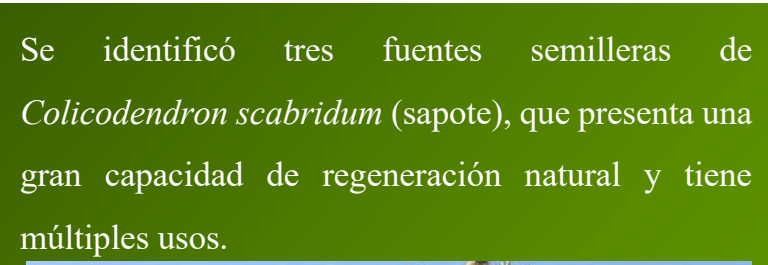
múltiples usos.

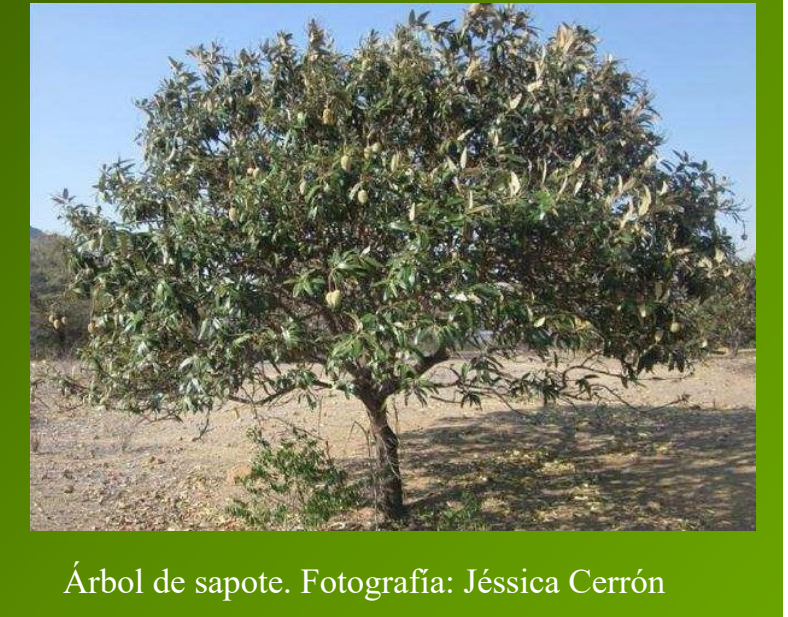
(Caesalpinia paipai), hualtaco (Loxopterygium huasango), almendro (Geoffroea spinosa) y pasallo (Eriotheca ruizii), que fueron identificados anteriormente en el marco de un proyecto específico $^{2}$, con excepción del charán. Asimismo, en el caso del período de 3 a 5 años, se identificó solo una fuente semillera para las otras especies (Figura 3).

\footnotetext{
${ }^{2}$ Proyecto: Fortalecimiento de las capacidades locales para la producción de semillas de seis especies forestales de los bosques secos de la región Piura (Universidad de Piura y Centro Ideas 2003)
} 


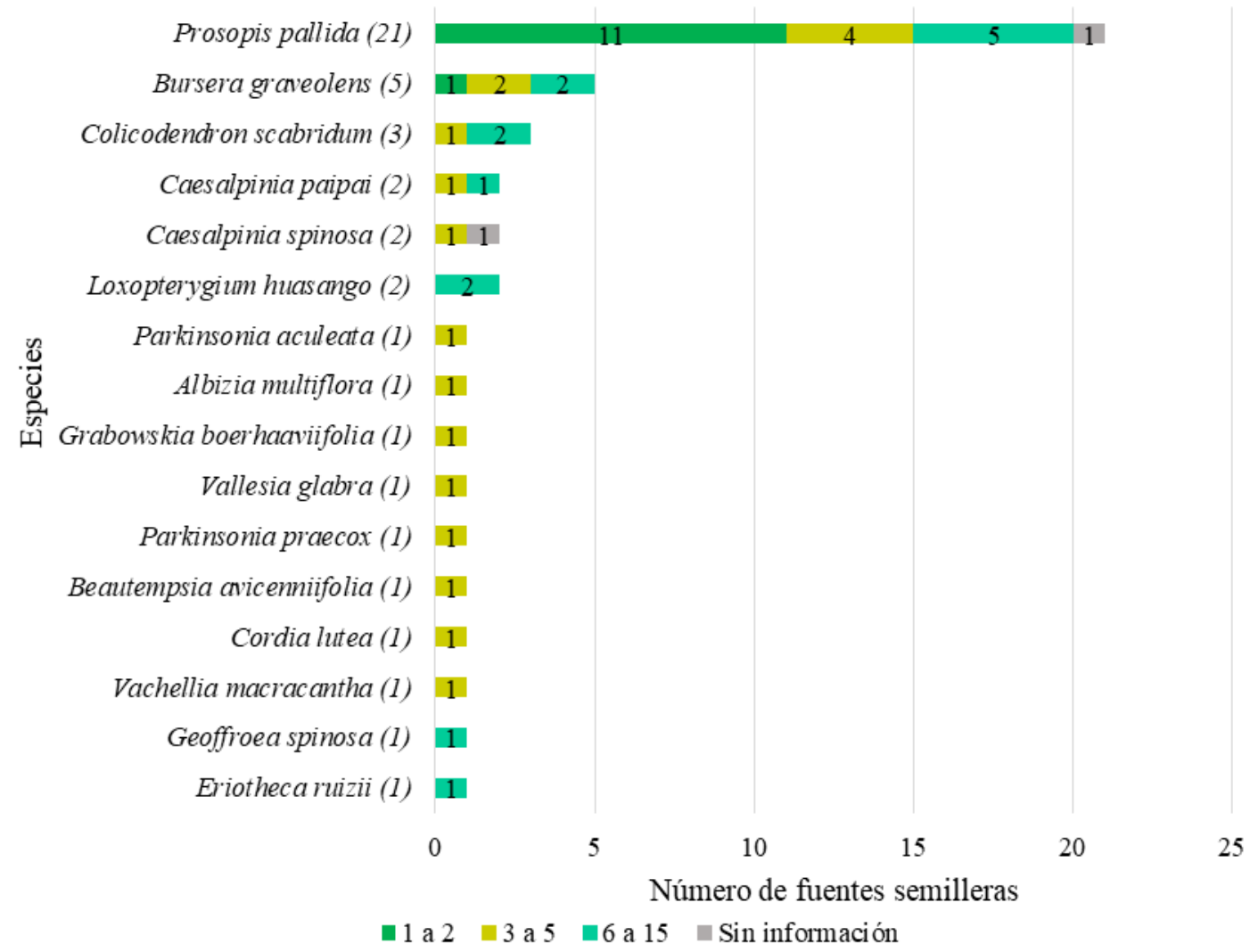

Figura 3. Número de fuentes semilleras por especie y período desde su establecimiento ( $N=45)$

\section{Tamaño poblacional y número de árboles seleccionados $(\mathrm{n}=\mathbf{2 1})$}

De las 45 fuentes semilleras existentes, solo 21 de ellas presentaron información del tamaño poblacional y cantidad de árboles seleccionados (semilleros), cuya distribución se aprecia en la Figura 4, donde predominó el tamaño poblacional de >300 árboles con 11 fuentes, seguido del rango de 101 a 300 con nueve fuentes, por último los rangos de 51 a 100 y <50 árboles con una fuente respectivamente.. Asimismo, en el caso del número de árboles seleccionados se observa la predominancia del rango de 21 a 40 con cinco fuentes, donde destacó el algarrobo con cuatro de éstas. Sin embargo, cabe resaltar que no se tiene información de la producción de semillas de éstos, por ello sería pertinente realizar un estudio más detallado de dicha variable. 


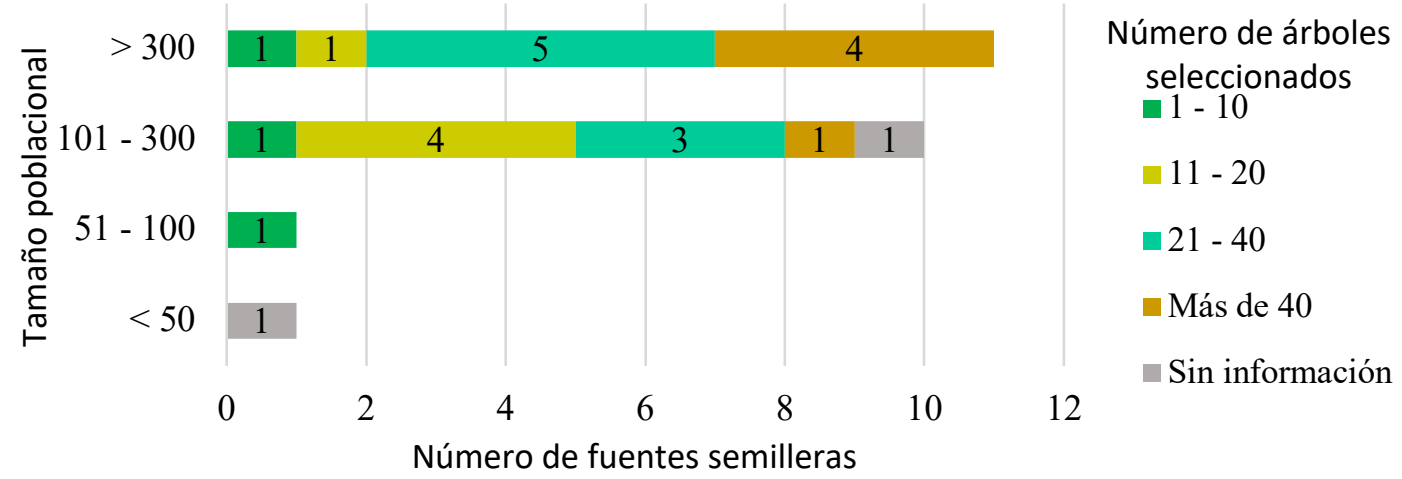

Figura 4. Número de fuentes semilleras por tamaño poblacional y número de árboles seleccionados

$$
(n=21)
$$

\section{Densidad (árboles/ha) y tipo de fuente de semillas ( $n=24)$}

De las 45 fuentes semilleras existentes, solo 24 de éstas presentaron información de densidad por hectárea, a partir de la cual se determinó el tipo de fuente semillera (Figura 5), predominando la categoría "fuente identificada" con 21 fuentes, seguido de la categoría "fuente seleccionada" con tres. De otro lado, el material reproductivo extraído es principalmente solo semillas ( 36 de 45 ) y de las nueves restantes se extraen tanto semillas como estacas, la cual está relacionado con la facilidad de propagación vegetativa a partir de estacas de las siguientes especies: palo santo (Bursera graveolens), hualtaco (Loxopterygium huasango), overo (Cordia lutea), cuncun (Vallesia glabra) y canutillo (Grabowskia boerhaaviifolia).

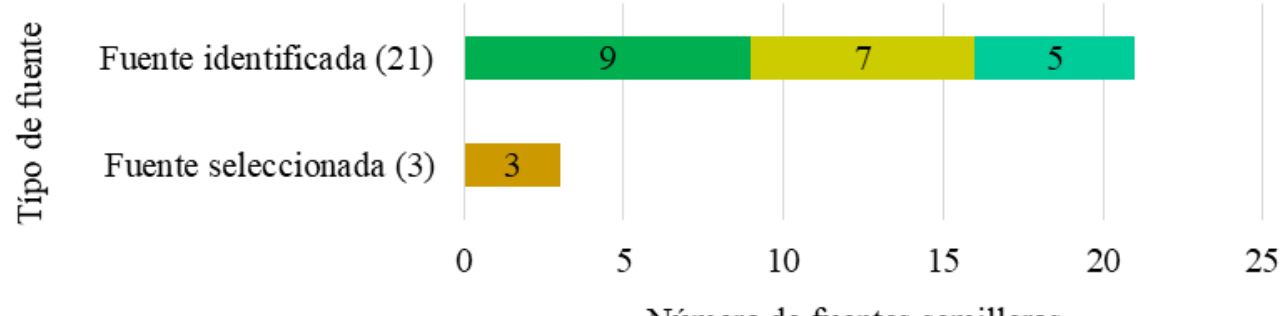

Número de fuentes semilleras

$$
\text { ๘ } 1 \text { - } 50 \text { árb/ha } \square 51-100 \square 101-200 \text { Más de } 200
$$

Figura 5. Densidad y tipo de fuente semillera $(n=24)$ 


\section{Distribución de fuentes semilleras potenciales ( $N=27)$}

A partir del mapeo realizado, se logró determinar 27 fuentes semilleras potenciales de 324 especies (Anexo 4) en el contexto de áreas de conservación nacional, regional y privada, entendiéndose el término "potencial", como áreas con presencia de especies leñosas maduras de individuos con características fenotípicas deseables para ser considerados semilleros; sin embargo, según los actores locales éstas aún no han sido identificadas para tal propósito. En relación a ello, en la Figura 6 se aprecia la distribución de éstas posibles fuentes, siendo preliminarmente Piura la de mayor proporción al contar con 12 áreas de conservación, seguido de Lambayeque, Tumbes y en menor proporción Cajamarca, Amazonas y La Libertad.

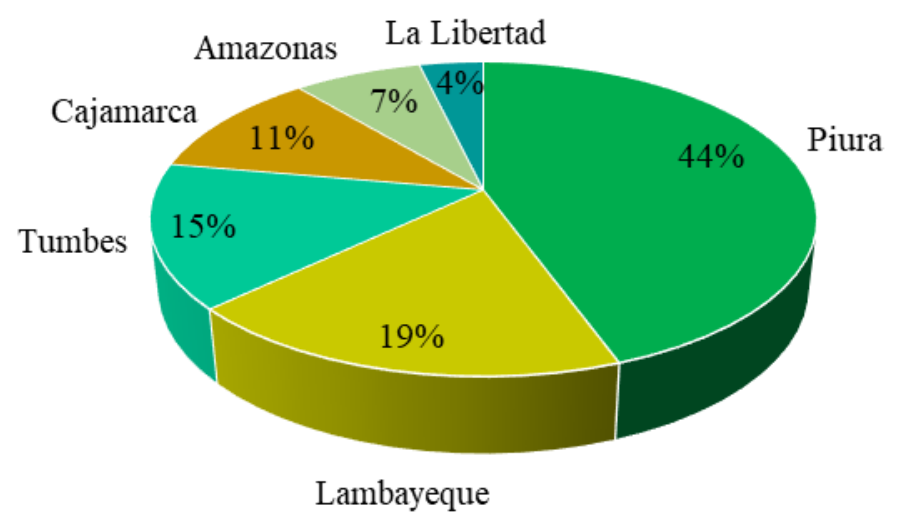

Figura 6. Distribución de fuentes semilleras potenciales $(\mathrm{N}=27)$

\section{Especies forestales en estado de conservación o amenaza}

\section{Bosques secos de Tumbes-Piura}

En el Anexo 5 se aprecia el estado de conservación y/o amenaza del total de especies determinadas para los bosques secos de Tumbes-Piura. En la Tabla 1 se presenta solo las especies que presentan algún estado de amenaza. 
Tabla 1. Especies de los bosques secos de Tumbes-Piura en estado de amenaza

\begin{tabular}{|l|l|}
\hline Fuente & Estado de conservación y/o amenaza de especies \\
\hline IUCN (2018) & $\begin{array}{l}\text { Preocupación Menor: Alnus acuminata (aliso), Caesalpinia } \\
\text { paipai (charán), Geoffroea spinosa (almendro) y Vallesia } \\
\text { glabra (cuncun) }\end{array}$ \\
\hline Apéndices CITES (2016) & Ninguna \\
\hline Resolución Ministerial 0505- & $\begin{array}{l}\text { Peligro crítico (CR): Bursera graveolens } \\
\text { (palo santo), Loxopterygium huasango(hualtaco) } \\
\text { En peligro (EN): Colicodendron scabridum } \\
\text { (sapote) y Prosopis pallida (algarrobo) } \\
\text { Vulnerable (VU): Beautempsia avicenniifolia (vichayo), } \\
\text { Grabowskia boerhaaviifolia (canutillo) y Vachellia } \\
\text { macracantha (faique) } \\
\text { Casi amenazado (NT): Alnus acuminata (aliso) }\end{array}$ \\
\hline
\end{tabular}

De otro lado, las especies del bosque seco de Tumbes-Piura en estado de amenaza citados por expertos locales se presentan la Tabla 2, clasificadas en función a los principales factores que influyen en su estado actual. 
Tabla 2. Especies de los bosque secos de Tumbes-Piura en estado de amenaza según expertos locales

\begin{tabular}{|c|c|c|c|c|c|c|}
\hline \multirow[b]{2}{*}{ Especies citadas por expertos locales } & \multicolumn{6}{|c|}{ Factores influyentes del estado actual de amenaza de especies citadas } \\
\hline & $\begin{array}{l}\text { Aprovechamiento } \\
\text { de su madera por } \\
\text { su valor } \\
\text { comercial }\end{array}$ & \begin{tabular}{|} 
Distribución \\
geográfica \\
restringida
\end{tabular} & $\begin{array}{c}\text { Susceptibilidad } \\
\text { a la sequía } \\
\text { prolongada } \\
\text { que afecta } \\
\text { floración }\end{array}$ & $\begin{array}{c}\text { Regeneración } \\
\text { natural no } \\
\text { abundante }\end{array}$ & $\begin{array}{c}\text { Alimento } \\
\text { y hábitat } \\
\text { de fauna } \\
\text { específica }\end{array}$ & $\begin{array}{c}\text { Usos } \\
\text { múltiples } \\
\text { para } \\
\text { beneficio } \\
\text { de los } \\
\text { pobladores }\end{array}$ \\
\hline Albizia multiflora (angolo) & & & & & & $\mathrm{x}$ \\
\hline Bursera graveolens (palo santo) & $x$ & & $x$ & & & $\mathrm{x}$ \\
\hline Caesalpinia paipai (charán) & & $x$ & & & & $\mathrm{x}$ \\
\hline Ceiba trischistandra (ceibo) & & $x$ & & & & $\mathrm{x}$ \\
\hline Celtis loxensis (palo blanco) & $\mathrm{x}$ & $\mathrm{x}$ & & & $x$ & \\
\hline Colicodendron scabridum (sapote) & $x$ & & & & $x$ & $\mathrm{x}$ \\
\hline Cordia lutea (overo) & & & & & $\mathrm{x}$ & $\mathrm{x}$ \\
\hline Eriotheca ruizii (pasallo) & & $\mathrm{x}$ & & & $x$ & $x$ \\
\hline Ficus nymphaeifolia (higuerón) & & & & & $x$ & \\
\hline Geoffroea spinosa (almendro de pepa) & & & & $x$ & & \\
\hline Grabowskia boerhaaviifolia (canutillo) & & $x$ & & & $x$ & \\
\hline Handroanthus billbergii (guayacán) & $x$ & & $x$ & $\mathrm{x}$ & & \\
\hline Handroanthus chrysanthus (guayacán) & $x$ & & $x$ & & & \\
\hline Leucaena trichodes (shapa) & & & & & $x$ & \\
\hline Loxopterygium huasango (hualtaco) & $x$ & $x$ & $x$ & $x$ & & \\
\hline Maclura tinctoria (sota, chamelico) & $x$ & & & & & $\mathrm{x}$ \\
\hline Piscidia carthagenensis (barbasco) & $x$ & & & & & \\
\hline Pithecellobium excelsum (chaquirón) & & & & & $x$ & \\
\hline Prosopis pallida (algarrobo) & $\mathrm{x}$ & & $x$ & & $x$ & $x$ \\
\hline Terminalia valverdeae (huarapo) & $x$ & & & & & \\
\hline Vachellia macracantha (faique) & $x$ & & & & & $x$ \\
\hline Ziziphus thirsiflora (ébano) & $x$ & & & & & \\
\hline
\end{tabular}

*Los datos de contacto de los expertos locales entrevistados se encuentran en el Anexo 6.

Esta lista de especies fue complementada por Tobías Fremout a partir del estudio que viene desarrollando.

En relación a las especies citadas por los expertos locales para la ecorregión bosques secos de Tumbes-Piura, en el caso del algarrobo (Prosopis pallida) durante las visitas en campo se observó la mortandad de individuos adultos y jóvenes principalmente en Lambayeque y en menor proporción en Piura. Al respecto, SENASA (2016) menciona que la mayor cantidad de estas muertes se da en los departamentos de Piura y Lambayeque y en menor grado en Tumbes, donde el porcentaje de muerte de los árboles de algarrobo va del 30 al 100\% de los individuos en las diferentes zonas que conforman el bosque seco, es decir hay poblaciones de árboles muertos, así como zonas en donde existen poblaciones de árboles en situación regular. En dicho contexto, diversas investigaciones se han realizado para conocer los factores que vienen influyendo en la mortandad de ésta especie, entre ellas las evaluaciones 
fitosanitarias entre setiembre de 2014 y 2016, que permitieron conocer que las plagas que afectan a Prosopis pallida en los bosques secos del norte del país son plagas que por su biología y daño que causan naturalmente, no podrían causar, individualmente, la muerte de los árboles en las cantidades que se vienen dando actualmente. Posiblemente la interacción de varias de estas plagas sea uno de los factores por el cual los bosques no logren desarrollar adecuadamente, aunado a las extremas condiciones ambientales en la que se desarrollan los árboles. Se podría especular que el cambio climático está favoreciendo la proliferación de hongos patógenos y plagas insectiles que se alimentan de los árboles, disminuyendo su floración y fructificación y posteriormente matándola, sumándose a ello el efecto drástico de los años de sequía prolongada la cual limita el crecimiento de nuevas poblaciones (Llontop 2013, Whaley et al. 2010, SENASA 2016).

Con respecto al sapote (Colicodendron scabridum) se observó su gran capacidad de regeneración natural y la ausencia de individuos con diámetros mayores a $10 \mathrm{~cm}$ en algunas zonas de Lambayeque (Mórrope y Ferreñafe) y Piura (Morropón y Paita); se considera que esto se debe a la utilización de la especie para leña, carbón y artesanías (Cabrejos et al. 2006 y Albán 2005).

De otro lado, en el distrito Salitral de Piura se observó individuos adultos con diámetros mayores a $20 \mathrm{~cm}$ de palo santo (Bursera graveolens) y hualtaco (Loxopterygium huasango), que se atribuye a que se encuentran en un Área de Conservación Privada, lo cual limita su aprovechamiento. Asimismo, según Albán (2005) el número de árboles por ha de Bursera graveolens y Loxopterygium huasango se ha reducido en $75 \%$ y $40 \%$ respectivamente en Piura producto de la tala selectiva, lo que representa un peligro para su sobrevivencia; además, Cabrejos et al. (2006) mencionan que Loxopterygium huasango no tiene gran capacidad de regeneración natural y en el caso de Bursera graveolens, la regeneración natural está limitada a periodos lluviosos.

En relación a las especies guayacán (Handroanthus billbergii) y polo polo (Cochlospermum vitifolium), no se llegaron a observar en campo; sin embargo, Odar (2010) registró los valores más bajos de densidad en los bosques secos del Coto de caza "El Angolo" ${ }^{3}$, concluyendo que puede deberse a que son especies altamente explotadas por su valor comercial y que presentan una distribución restringida. Con respecto a ello, Cabrejos et al. (2006) mencionan que Cochlospermum vitifolium se ubica en bosques secos semidensos de montaña y bosques secos semidensos de colina y Albán (2005) resalta que una muestra representativa de Handroanthus billbergii se encuentra en Piura, distrito de Suyo (Comunidad Campesina de Pampa Larga).

\footnotetext{
${ }^{3}$ Área Natural Protegida con categoría de Coto de caza ubicado en Piura, cuyo objetivo es la conservación del bosque seco ecuatorial y el manejo sostenible de la fauna silvestre.
} 
En el caso del overo (Cordia lutea) se observó una gran capacidad de regeneración natural, siendo utilizada por los pobladores como cercos perimétricos y cercos para protección de regeneración natural de algarrobo y sapote; por lo tanto, no se aprecia un uso desmedido de la especie para categorizarla como amenazada. Al respecto, Cabrejos et al. (2006) mencionan que esta especie se encuentra distribuida en bosques secos de llanura, bosques secos de colina y es usado para confeccionar mangos de herramientas, muebles, barriles, postes para cercos de predios y como leña.

De otro lado, en el caso del almendro de pepa (Geoffroea spinosa), Cabrejos et al. (2006) resaltan que es una especie de interés comercial por tener un veteado particular y por ello es extraída desmesuradamente por la población; además, mencionan que presenta regeneración natural no abundante y forma parte de los bosques secos semidensos de montaña y bosques secos semidensos de colina. Los mismos autores mencionan que la madera de charán (Caesalpinia paipai) es extraída para usos como la carpintería, leña y carbón y que se encuentra formando parte de diferentes tipos de bosques secos desde semidensos y ralos en colinas, lomadas y montañas.

En relación al faique (Vachellia macracantha) se observaron pocos individuos con diámetros mayores a $15 \mathrm{~cm}$, lo cual según comuneros de la zona es debido a que se han ido extrayendo los individuos adultos para hacer uso de su madera. Cabrejos et al. (2006) mencionan que esta especie se distribuye en la faja costera y forma parte de los bosques secos semidensos de montaña, bosques seco ralo-muy ralo de montaña, entre otros y es altamente extraída para usarla como material para embarcaciones artesanales, leña, carbón y fabricación de mangos de herramientas.

De otro lado, se observaron algunos individuos jóvenes de las especies: palo blanco (Celtis loxensis), higuerón (Ficus nymphaeifolia), pasallo (Eriotheca ruizii), chaquirón (Pithecellobium excelsum) y shapa (Leucaena trichodes) de manera dispersa en el Refugio de Vida Silvestre de Laquipampa que, según el jefe del área, son plantones que fueron sembrados en el refugio con el fin de mejorar la conectividad ecológica de la pava aliblanca (Penelope albipensis), ave que se encuentra en peligro crítico según D.S. N004-2014-MINAGRI, ya que forma parte de su hábitat y dieta alimenticia. En relación a Celtis loxensis Cabrejos et al. (2006) mencionan que esta especie se encuentra distribuida en bosques semidensos de montaña y bosques semidensos de colina y es extraída para usarla en cajonería y artesanías.

Con respecto a la especie canutillo (Grabowskia boerhaaviifolia), Lazo (2014) menciona que en los bosques del Santuario Histórico de Pómac existe solo un $10 \%$ de cobertura de esta especie y resalta su importancia ya que no solo es parte de la dieta alimenticia sino también garantiza el éxito reproductivo de la cortarrama peruana (Phytotoma raimondii), ave que se encuentra catalogada "en peligro" según el D.S. N004-2014-MINAGRI. Por último, el ceibo (Ceiba trischistandra) según Castro (2007) se encuentra presionada por la ampliación de la 
frontera agrícola y es considerada endémica de la denominada "región tumbesina" que comprende el occidente del Ecuador y el noroeste del Perú (DarwinNet 2005, citado por Castro 2007), formando parte del bosque seco de colina y bosque seco de montaña (INRENA 1995, citado por Castro 2007). Presenta múltiples usos a partir de la madera, corteza, semillas y fibra denominada "kapok", ésta última es un producto con gran potencial comercial, pero con deficiente promoción y mercado en el Perú.

\section{Bosques secos del Marañon}

En el Anexo 7 se presenta la lista completa de las especies leñosas de interés para su conservación que deberían ser tomadas en cuenta, basada en su estado de amenaza y endemismo. En la Tabla 3 se presenta solo las especies en algún estado de amenaza.

Tabla 3. Especies del bosque seco del Marañon en estado de amenaza

\begin{tabular}{|l|l|}
\hline Fuente & Especies en estado de conservación y/o amenaza \\
\hline IUCN (2018) & $\begin{array}{l}\text { Peligro crítico: Parkinsonia peruviana - En peligro: Aspidosperma polyneuron } \\
\text { Vulnerable: Bauhinia augusti y Piptadenia weberbaueri } \\
\text { Preocupación menor: Geoffroea spinosa }\end{array}$ \\
\hline $\begin{array}{l}\text { Resolución } \\
\text { Ministerial 0505- }\end{array}$ & $\begin{array}{l}\text { En peligro (EN): Prosopis juliflora } \\
\text { Vulnerable (VU): Vachellia macracantha }\end{array}$ \\
\hline $\begin{array}{l}\text { Marcelo-Peña et al. } \\
\text { (2010)* }\end{array}$ & $\begin{array}{l}\text { Peligro crítico: Esenbeckia cornuta, Maraniona lavinii y Pseudobombax } \\
\text { cajamarcanus } \\
\text { incarum y Tetrasida serrulata } \\
\text { Vulnerable: Cordia iguaguana, Coursetia maraniona, Jatropha humboldtiana, } \\
\text { Ruprechtia aperta, Tecoma rosifolia y Tetrasida chachapoyensis } \\
\text { Preocupación menor: Mimosa pectinatipinna }\end{array}$ \\
\hline
\end{tabular}

*Marcelo-Peña et al. (2010) categorizan a partir de sus observaciones en campo.

De otro lado, en el caso de las especies endémicas, éstas se presentan en la Tabla 4. 
Tabla 4. Especies leñosas endémicas de los bosques secos del Marañon

\begin{tabular}{|l|l|l|}
\hline León et al. (2006) & Marcelo Peña et al. (2010) & Marcelo Peña et al. (2015) \\
\hline Abutilon pedunculare & Bauhinia augusti & Abutilon pedunculare \\
Albizia multiflora & Cordia iguaguana & Allamanda weberbaueri \\
Allamanda weberbaueri & Cordia varronifolia & Bauhinia augusti \\
Aristolochia chachapoyensis & Coursetia maraniona & Cordia iguaguana \\
Aristolochia xerophytica & Croton adipatus & Cordia varronifolia \\
Cordia iguaguana & Esenbeckia cornuta & Coursetia maraniona \\
Cordia varronifolia & Jatropha humboldtiana & Croton adipatus \\
Coursetia maraniona & Maraniona lavinii & Croton rubiginosus \\
Croton adipatus & Mimosa incarum & Esenbeckia cornuta \\
Croton rubiginosus & Mimosa pectinatipinna & Jatropha humboldtiana \\
Esenbeckia cornuta & Pseudobombax cajamarcanus & Maraniona lavinii \\
Jatropha humboldtiana & Ruprechtia aperta & Mimosa incarum \\
Maraniona lavinii & Tecoma rosifolia & Mimosa pectinatipinna \\
Mimosa incarum & Tetrasida chachapoyensis & Parkinsonia peruviana \\
Mimosa pectinatipinna & Tetrasida serrulata & Piptadenia weberbaueri \\
Parkinsonia peruviana & & Pseudobombax \\
Piptadenia weberbaueri & & cajamarcanus \\
Ruprechtia aperta & & Ruprechtia aperta \\
Tetrasida chachapoyensis & & Tecoma rosifolia \\
Tetrasida serrulata & & Tetrasida chachapoyensis \\
& & Tetrasida serrulata \\
\hline
\end{tabular}

En el caso del bosque seco del Marañon, no se identificó actores locales claves con suficiente conocimiento de las especies; sin embargo, a partir de la revisión bibliográfica del estudio de composición florística realizado por Marcelo-Peña et al. (2010) se logró determinar una lista de especies de interés por su uso bajo diversas prácticas agroforestales (Tabla 5), que en algunos casos cuentan con categoría de amenaza y/o endemismo (ver detalle en Anexo 7). 
Tabla 5. Especies leñosas de los bosques secos del Marañon con uso agroforestal

\begin{tabular}{|l|l|}
\hline \multicolumn{1}{|c|}{ Nombre científico } & \multicolumn{1}{c|}{ Usos en prácticas agroforestales } \\
\hline Acacia aroma & Sistemas silvopastoriles, sombra \\
\hline Albizia multiflora & Sistemas silvopastoriles, sombra \\
\hline Albizia niopoides & Sombra \\
\hline Anadenanthera colubrina & Sistemas agroforestales y silvopastoriles \\
\hline Aspidosperma polyneuron & Sistemas silvopastoriles, sombra \\
\hline Cordia iguaguana & Sistemas silvopastoriles, sombra \\
\hline Cyathostegia matthewsii & Cercos vivos \\
\hline Geoffroea spinosa & Sombra, defensa ribereña \\
\hline Guazuma ulmifolia & Sistemas silvopastoriles, sombra \\
\hline Leucaena trichodes & Sombra de café \\
\hline Mimosa incarum & Setos vivos, fijación de taludes \\
\hline Mimosa pectinatipinna & Estabilización de taludes \\
\hline Muntingia calabura & Fijación de taludes \\
\hline Prosopis juliflora & Sistemas silvopastoriles, sombra \\
\hline Sapindus saponaria & Sistemas silvopastoriles, sombra, cercos \\
\hline Senegalia polyphylla & Sombra \\
\hline Senna mollissima & Sombra \\
\hline Sideroxylon obtusifolium & Sistemas silvopastoriles, sombra \\
\hline Tecoma rosifolia & Estabilización de taludes \\
\hline Tessaria integrifolia & Defensa ribereña \\
\hline Vachellia macracantha & Sistemas silvopastoriles, sombra \\
\hline
\end{tabular}




\section{Conclusiones}

Se identificaron 45 fuentes semilleras de 16 especies en la ecorregión bosques secos de Tumbes-Piura y ninguna en bosques secos del Marañon; sin embargo, existen 27 fuentes semilleras potenciales de 324 especies en las áreas de conservación.

Las fuentes semilleras existentes se encuentran principalmente bajo propiedad de las comunidades campesinas y en menor porcentaje en predios privados, asociaciones locales y entidades estatales.

Predominaron las fuentes semilleras de algarrobo (Prosopis spp.) con $47 \%$ y palo santo (Bursera graveolens) con $11 \%$ debido a sus usos e interés comercial y en menor cantidad de otras especies, siendo el tamaño poblacional predominante mayor a 300 árboles y el número de árboles seleccionados como semilleros de 21 a 40 individuos.

Las fuentes semilleras existentes son en su mayoría fuentes identificadas en función a su densidad y características de selección. No se encontraron huertos y rodales semilleros.

En los bosques secos de Tumbes-Piura se identificaron preliminarmente 11 especies bajo diferentes categorías de conservación o amenaza de las clasificaciones oficiales. Asimismo, 22 especies fueron identificadas bajo amenaza por expertos locales, de los cuales siete de éstas, que son utilizadas para proyectos de reforestación se encuentran en alguna categoría de amenaza oficial.

En los bosques secos del Marañon se identificaron preliminarmente siete especies bajo diferentes categorías de conservación o amenaza de las clasificaciones oficiales. Asimismo, 21 especies fueron identificadas de interés potencial por su uso bajo diversas prácticas agroforestales, de las cualesnueve se encuentran en alguna categoría de amenaza oficial y seis son endémicas.

\section{Recomendaciones}

Es importante tener en cuenta el nivel de interés de las comunidades campesinas para el establecimiento y mantenimiento de fuentes semilleras, por ello es indispensable conocer el estado actual y el interés potencial de una red de actores para la compra y venta de semillas forestales en los bosques secos. Preliminarmente, algunos actores locales coinciden en señalar que no existe un mercado formal de oferta y demanda de semillas forestales y que la comercialización de éstas no sería un negocio rentable a menos que se encuentre una 
estrategia atractiva para la población local. Cabe resaltar que lo que predomina en el mercado actual es la producción y venta de plantones, especialmente de algarrobo ya que de las otras especies existen en mínimas cantidades y solo se activan temporalmente en función a proyectos o programas especificos del estado o del sector privado.

Si bien existe disponibilidad de área de bosque seco con especies de interés para el establecimiento de fuentes semilleras, es indispensable seleccionar las zonas a priorizar considerando el nivel de organización local de las comunidades campesinas que puedan comprometerse con el manejo de éstas a largo plazo, lo cual garantice su sostenibilidad. En dicho contexto, se sugiere preliminarmente la articulación entre las comunidades campesinas que ya tienen identificado fuentes semilleras de especies representativas del bosque seco de Ilanura (Prosopis pallida y Colicodendron scabridum) y del bosque seco de colina (Loxopterygium huasango, Bursera graveolens, Eriotheca ruizii y Geoffroea spinosa) para ser partícipes de actividades de fortalecimiento de capacidades y registro de sus fuentes.

De otro lado, a pesar de la variedad de especies en el bosque seco del Marañon con alto potencial para reforestación en zonas áridas, usos bajo prácticas agroforestales y como estabilizadoras de suelos, no hay registro de fuentes semilleras de éstas especies. Por lo tanto, se sugiere primero priorizar una lista de interés contrastando el estado de amenaza, endemismo, usos, comportamiento fenológico e información de métodos de propagación de dichas especies y posteriormente realizar un mapeo de la disponibilidad y producción de semillas para planificar futuras actividades.

Para la priorización de especies se sugiere no solo contrastar la información del estado de amenaza y/o conservación con la demanda de éstas especies para proyectos de reforestación, recuperación y restauración u otros, sino también la disponibilidad y producción de semillas $\mathrm{u}$ otros materiales de siembra de las especies seleccionadas enun contexto de cambio climático.

Los bosques secos tropicales del norte del Perú presentan una variedad de especies; sin embargo, existe la tala selectiva de solo algunas especies tales como: Prosopis pallida, Colicodendron scabridum, Vachellia macracantha, Bursera graveolens, Handroanthus billbergi y $H$. chrysanthus por su potencial maderable de gran rentabilidad a diferencia de otras especies, siendo extraídos de los bosques secos de forma ilegal y solo en algunos casos en el marco de planes de manejo. Por ende, hay una gran controversia entre lo extraído y lo reforestado, ya que la mayoría de proyectos se enfocan principalmente en Prosopis pallida, Bursera graveolens y en menor proporción Colicodendron scabridum, dejando de lado las otras especies; debido, según actores locales, principalmente a la deficiencia de material reproductivo y de conocimientos de propagación de éstas. Sería oportuno, en la medida posible realizar un mapeo de las principales deficiencias de conocimientos técnicos de 
colección, almacenamiento y propagación de las especies de interés, que permita abordar la temática de forma más dinámica y planificar futuras actividades. 


\section{Anexos}

Anexo 1. Formulario de fuentes semilleras en áreas de conservación

\section{Nombre del área}

1.1 Código: (Ej. CPIU1)

1.2 Título:

2. Características del área

2.1 ¿Cuándo fue declarada como área de conservación?

2.2 ¿Qué tipo de administración presenta el área?, y de ser posible mencionar el nombre de la persona o entidad administradora

() Áreas de Administración Nacional

() Áreas de Conservación Regional

() Áreas de Conservación Privada

Nombre del administrador:

Nombre del responsable:

Correo electrónico:

Teléfono fijo o móvil:

2.3 ¿Dónde se encuentra localizada y cuál es su extensión?
- Departamento:
- Provincia:
- Distrito:
- Localidad, caserío u otro:
- Coordenadas geográficas y/o UTM:
- Datum:
- Extensión (ha):

2.4 ¿Existe alguna lista de especies de flora presentes en el área de conservación?
() $\mathrm{Si}$
() No

2.5 En caso hubiese la lista de especies, ¿Con qué variables cuenta? (Puede marcar más de una)
( ) Altura
( ) Diámetro
( ) Densidad o abundancia relativa
() Frecuencia
() Dominancia
() Estado fitosanitario
( ) Coordenadas
() Ninguna
() Otros (especifique):

3. Características de las fuentes semilleras

3.1 ¿Se tienen identificados fuentes semilleras de éstas especies? Especificar para qué especie (s)
() $\mathrm{Si}$
() No

3.2 ¿Dónde se encuentran localizadas las fuentes semilleras? Especificar para cada especie
- Coordenadas: Latitud. Longitud Datum
- Altitud:

3.3 Características de la fuente semillera para cada especie

- Fecha de establecimiento o identificación:

- Período desde su establecimiento:

- Superficie (ha):

○ Densidad (árb/ha): 

- Tamaño poblacional:
- Número de árboles seleccionados:

3.2 ¿Qué criterios se utilizaron para identificarlas? Especificar para cada especie

() Accesibilidad y topografía

( ) Condición del rodal (estado fitosanitario, capacidad de producir semillas)

( ) Número de árboles y tamaño de fuente

() Edad de los árboles

() Especies únicas

() Ninguna

() Otros (especifique):

3.3 ¿Qué tipo de fuente semillera es? Especificar para cada especie

() Rodal semillero a partir de plantación existente (natural)

() Rodal semillero a partir de una nueva plantación (establecido)

() Fuente seleccionada

() Fuente identificada

3.4 En las fuentes semilleras, ¿Qué características se tomaron en cuenta para identificar los árboles plus? (Puede marcar más de una) Especificar para cada especie
( ) Fustes rectos
() Forma de copa regular
() Ausencia de bifurcaciones en la base
() Estado fitosanitario bueno
() Ser dominante (excepcionalmente codominante)
() Ninguna
() Otros (especifique):

3.5 ¿Qué tipo de material reproductivo se extraen de las fuentes semilleras? Especificar para cada especie

() Semillas

() Estacas

() Otros (especifique): 
Anexo 2. Formulario de fuentes semilleras en otras zonas

\section{Datos generales}

1.1 Código: (Ej. SPIU1)

1.2 Nombre de la fuente semillera:

1.3 ¿A quién le pertenece la fuente semillera? De ser posible, mencionar su nombre

() Comunidad local

() Asociación

() Empresa privada

() ONG

() Centro de investigación

() Universidad

() Otro (especifique)

Nombre del propietario:

Nombre del responsable:

Correo electrónico:

Teléfono fijo o móvil:

1.4 ¿Dónde se encuentra localizada y cuál es su superficie?

- Departamento:

- Provincia:

- Distrito:

- Localidad, caserío u otro:

- Altitud (msnm):

- Coordenadas geográficas y/o UTM:

- Datum:

- Superficie (ha):

- Superficie total del PGMF* (ha):

2. Características de las fuentes semilleras

2.1 Información técnica de la (s) especies. Especificar para cada especie

- Nombre científico:

- Nombre común:

- Fecha de establecimiento o identificación:

- Período de identificación:

- Tamaño poblacional:

○ Densidad (árb/ha):

- Número de árboles seleccionados:

2.2 ¿Qué criterios se utilizaron para identificar las fuentes semilleras? Especificar para cada especie

() Accesibilidad y topografía

( ) Condición del rodal (estado fitosanitario, capacidad de producir semillas)

( ) Número de árboles y tamaño de fuente

() Edad de los árboles

() Especies únicas

() Ninguna

() Otros (especifique):

2.3 ¿Qué tipo de fuente semillera es? Especificar para cada especie

() Huerto semillero forestal comprobado

() Huerto semillero forestal no comprobado

() Rodal semillero a partir de plantación existente (natural)

() Rodal semillero a partir de una nueva plantación (establecido) 
( ) Fuente seleccionada

() Fuente identificada

2.4 En las fuentes semilleras, ¿Qué características se tomaron en cuenta para identificar los árboles plus? (Puede marcar más de una) Especificar para cada especie

( ) Fustes rectos

( ) Forma de copa regular

() Ausencia de bifurcaciones en la base

() Estado fitosanitario bueno

( ) Ser dominante (excepcionalmente codominante)

( ) Ninguna

() Otros (especifique):

2.5 ¿Qué tipo de material reproductivo se extraen de las fuentes semilleras? Especificar para cada especie

( ) Semillas

() Estacas

() Otros (especifique): 
Anexo 3. Categorías de fuentes semilleras

FUENTE SEMILLERA: Según MINAGRI (2006) y Cuellar et al. (2016), son individuos seleccionados en base a características fenotípicas y genéticas deseables que proveen material reproductivo (generalmente semillas) de calidad. Las fuentes semilleras se clasifican en cinco categorías:

\begin{tabular}{|c|c|}
\hline Categoría & Características \\
\hline $\begin{array}{l}\text { Huerto } \\
\text { semillero } \\
\text { comprobado }\end{array}$ & $\begin{array}{l}\text { Plantación de clones o progenies seleccionadas intensivamente, aislada para reducir la } \\
\text { contaminación de polen de árboles inferiores y manejada intensivamente para la } \\
\text { producción de semillas. Tiene el respaldo de pruebas de progenies y ha sido sometido a } \\
\text { aclareos genéticos. }\end{array}$ \\
\hline $\begin{array}{l}\text { Huerto } \\
\text { semillero no } \\
\text { comprobado }\end{array}$ & $\begin{array}{l}\text { Huerto que no ha sido sometido a aclareos genéticos, ya sea por la ausencia de ensayos } \\
\text { genéticos o por la corta edad de los ensayos. Aunque este huerto no tiene el respaldo } \\
\text { de pruebas genéticas, la alta intensidad de selección a que han sido sometidos los } \\
\text { progenitores garantiza una ganancia genética superior a la de otros tipos de fuentes. }\end{array}$ \\
\hline $\begin{array}{l}\text { Rodal } \\
\text { semillero }\end{array}$ & $\begin{array}{l}\text { Pueden ser plantados o naturales, aislados o manejados para reducir la contaminación } \\
\text { de polen de árboles inferiores y que han sido sometidos a aclareos de mejoramiento } \\
\text { para dejar de } 75 \text { a } 200 \text { árboles por hectárea con características fenotípicas deseadas. El } \\
\text { rodal semillero deber tener un área mínima de } 1 \text { ha, grupos más pequeños o árboles en } \\
\text { hileras no pueden ser considerados en esta categoría. Una de las diferencias principales } \\
\text { entre rodales semilleros y huertos semilleros, es la intensidad de selección: en los } \\
\text { rodales semilleros los árboles han sido seleccionados a una intensidad de 1:10 - 1:20, } \\
\text { mientras que en el caso de los huertos, cada árbol ha sido seleccionado entre varios } \\
\text { miles de árboles evaluados. }\end{array}$ \\
\hline $\begin{array}{l}\text { Fuente } \\
\text { seleccionada }\end{array}$ & $\begin{array}{l}\text { Son fuentes que no cumplen con los requisitos establecidos para los rodales semilleros, } \\
\text { principalmente porque presentan problemas de aislamiento, y contienen menos de } 75 \\
\text { árboles aceptables por hectárea o porque no han sido sometidos a los aclareos de } \\
\text { depuración (contienen más de } 200 \text { árboles por hectárea). }\end{array}$ \\
\hline $\begin{array}{l}\text { Fuente } \\
\text { identificada }\end{array}$ & $\begin{array}{l}\text { Son grupos de árboles que por su baja densidad, por ocupar poca área y/o porque no } \\
\text { contienen el número suficiente de árboles con características deseables por hectárea, } \\
\text { no clasifican dentro de la categoría anterior, pero deben utilizarse temporalmente ante } \\
\text { la ausencia de fuentes más seleccionadas. En este grupo se encuentran típicamente: las } \\
\text { parcelas experimentales representadas por un número limitado de individuos, } \\
\text { pequeños bloques de plantación, ensayos genéticos o silviculturales de poca extensión, } \\
\text { especies del bosque natural que por su naturaleza o debido a la eliminación de bosques, } \\
\text { ocurren a bajas densidades o no alcanzan el número mínimo de árboles aceptables por } \\
\text { hectárea }\end{array}$ \\
\hline
\end{tabular}


Anexo 4. Lista de especies de fuentes semilleras potenciales

\begin{tabular}{|c|c|c|c|}
\hline \multicolumn{4}{|c|}{ Especies } \\
\hline Acalypha cuspidata & $\begin{array}{l}\text { Aspidosperma } \\
\text { polyneuron }\end{array}$ & Cariniana parvifolia & Coccoloba obovata \\
\hline Acalypha padifolia & Bauhinia aculeata & Casearia aculeata & $\begin{array}{l}\text { Coccoloba } \\
\text { padiformis }\end{array}$ \\
\hline Acalypha villosa & Bauhinia augusti & Casearia obovalis & Coccoloba ruiziana \\
\hline Acanthosyris glabrata & $\begin{array}{l}\text { Beautempsia } \\
\text { avicenniifolia } \\
\end{array}$ & $\begin{array}{l}\text { Cavanillesia } \\
\text { platanifolia }\end{array}$ & $\begin{array}{l}\text { Cochlospermum } \\
\text { vitifolium }\end{array}$ \\
\hline $\begin{array}{l}\text { Achatocarpus } \\
\text { pubescens }\end{array}$ & Bonellia mucronata & Cecropia garciae & Cocos nucifera \\
\hline Acnistus arborescens & Bonellia sprucei & $\begin{array}{l}\text { Cecropia } \\
\text { membranacea }\end{array}$ & \begin{tabular}{|l|}
$\begin{array}{l}\text { Colicodendron } \\
\text { scabridum }\end{array}$ \\
\end{tabular} \\
\hline $\begin{array}{l}\text { Aeschynomene } \\
\text { tumbezensis }\end{array}$ & $\begin{array}{l}\text { Bougainvillea } \\
\text { pachyphylla }\end{array}$ & Cedrela angustifolia & Cordia alliodora \\
\hline Agonandra excelsa & $\begin{array}{l}\text { Bougainvillea } \\
\text { peruviana }\end{array}$ & Cedrela fissilis & Cordia eriostigma \\
\hline Albizia guachapele & Briquetia spicata & Cedrela molinensis & Cordia iguaguana \\
\hline Albizia multiflora & Brosimum alicastrum & Ceiba insignis & Cordia lutea \\
\hline Albizia niopoides & Brugmansia versicolor & Ceiba trischistandra & Cordia panamensis \\
\hline Allophylus punctatus & Bunchosia plowmanii & Celtis iguanaea & Cordia saccellia \\
\hline Alnus acuminata & Bursera graveolens & Celtis loxensis & Cordia varronifolia \\
\hline $\begin{array}{l}\text { Aloysia } \\
\text { scorodonioides }\end{array}$ & Byttneria glabresens & Celtis schippii & Coursetia maraniona \\
\hline Alseis eggersii & Byttneria parviflora & \begin{tabular}{|l|}
$\begin{array}{l}\text { Centrolobium } \\
\text { ochroxylum }\end{array}$ \\
\end{tabular} & $\begin{array}{l}\text { Crescentia } \\
\text { amazonica }\end{array}$ \\
\hline Alseis peruviana & Caesalpinia cassioides & Cestrum auriculatum & $\begin{array}{l}\text { Critoniopsis } \\
\text { woytkowskii }\end{array}$ \\
\hline $\begin{array}{l}\text { Ampelocera } \\
\text { macphersonii }\end{array}$ & Caesalpinia glabrata & $\begin{array}{l}\begin{array}{l}\text { Chrysophyllum } \\
\text { cuneifolium }\end{array} \\
\end{array}$ & Croton adipatus \\
\hline Amyris pinnata & Caesalpinia spinosa & \begin{tabular}{|l|}
$\begin{array}{l}\text { Chrysophyllum } \\
\text { lucentifolium }\end{array}$ \\
\end{tabular} & Croton erythrochilus \\
\hline $\begin{array}{l}\text { Anadenanthera } \\
\text { colubrina }\end{array}$ & Calliandra angustifolia & Cinchona pubescens & Croton glabellus \\
\hline Annona montana & Calliandra mollissima & \begin{tabular}{|l|}
$\begin{array}{l}\text { Citharexylum } \\
\text { chartaceum }\end{array}$ \\
\end{tabular} & Croton schiedeanus \\
\hline Annona muricata & Callianthe geminiflora & Citharexylum gentryi & Croton thurifer \\
\hline Aphelandra attenuata & Callianthe pauciflora & Clavija euerganea & Cupania latifolia \\
\hline Aphelandra glabrata & $\begin{array}{l}\text { Capparicordis } \\
\text { crotonoides }\end{array}$ & Clusia elliptica & $\begin{array}{l}\text { Cyathostegia } \\
\text { mathewsii }\end{array}$ \\
\hline Aralia soratensis & $\begin{array}{l}\text { Capparidastrum } \\
\text { petiolare }\end{array}$ & Clusia peruviana & $\begin{array}{l}\text { Cybistax } \\
\text { antisyphilitica }\end{array}$ \\
\hline $\begin{array}{l}\text { Armatocereus } \\
\text { digogonus }\end{array}$ & Capsicum hookerianum & Cnidoscolus jaenensis & Cynophalla flexuosa \\
\hline
\end{tabular}


Continuación

\begin{tabular}{|c|c|c|c|}
\hline \multicolumn{4}{|c|}{ Especies } \\
\hline $\begin{array}{l}\text { Armatocereus } \\
\text { matucanensis }\end{array}$ & \begin{tabular}{|l|} 
Capsicum \\
rhomboideum
\end{tabular} & Coccoloba densifrons & $\begin{array}{l}\text { Cynophalla } \\
\text { heterophylla }\end{array}$ \\
\hline $\begin{array}{l}\text { Aspidosperma } \\
\text { excelsum }\end{array}$ & $\begin{array}{l}\text { Cardiospermum } \\
\text { halicacabum }\end{array}$ & Coccoloba mollis & $\begin{array}{l}\text { Cynophalla } \\
\text { sclerophylla }\end{array}$ \\
\hline Ditaxis dioica & Fridericia dichotoma & Krameria lappacea & Muntingia calabura \\
\hline Ditaxis katharinae & Gallesia integrifolia & $\begin{array}{l}\text { Ladenbergia } \\
\text { amazonensis }\end{array}$ & Myrcia splendens \\
\hline Drypetes amazonica & Geoffroea spinosa & Leucaena trichodes & Myrcianthes rhopaloides \\
\hline Duranta erecta & $\begin{array}{l}\text { Grabowskia } \\
\text { boerhaaviifolia }\end{array}$ & $\begin{array}{l}\text { Lonchocarpus } \\
\text { atropurpureus }\end{array}$ & Myroxylon balsamum \\
\hline Eriotheca discolor & Guazuma ulmifolia & $\begin{array}{l}\text { Lonchocarpus } \\
\text { floribundus }\end{array}$ & Myroxylon peruiferum \\
\hline Eriotheca ruizii & $\begin{array}{l}\text { Gymnosporia } \\
\text { emarginata }\end{array}$ & $\begin{array}{l}\text { Loxopterygium } \\
\text { huasango }\end{array}$ & Neea spruceana \\
\hline Erythrina smithiana & Hamelia patens & Luehea paniculata & Ochroma pyramidale \\
\hline Erythrina velutina & Handroanthus billbergii & Lycianthes stenoloba & Ocotea aurantiodora \\
\hline \begin{tabular}{|l|} 
Erythrochiton \\
odontoglossus \\
\end{tabular} & $\begin{array}{l}\text { Handroanthus } \\
\text { chrysanthus }\end{array}$ & Lysiloma latisiliquum & Ocotea piurensis \\
\hline Erythroxylum glaucum & Heliocarpus americanus & Machaerium millei & Pachira patinoi \\
\hline Erythroxylum pacificum & Heliotropium rufipilum & Maclura tinctoria & Pachira trinitensis \\
\hline Erythroxylum patens & Hilleria secunda & $\begin{array}{l}\text { Macrosamanea } \\
\text { amplissima }\end{array}$ & Parkinsonia aculeata \\
\hline Esenbeckia cornuta & Hura crepitans & Malpighia glabra & Parkinsonia praecox \\
\hline Eugenia biflora & Inga acrocephala & Malvaviscus arboreus & Phyllanthus mocinianus \\
\hline Eumachia microdon & Inga codonantha & Mansoa hymenaea & Phytolacca dioica \\
\hline Faramea capillipes & Inga feuillei & Maraniona lavinii & Phytolacca weberbaueri \\
\hline Faramea occidentalis & Inga ruiziana & Margaritaria nobilis & Picramnia sellowii \\
\hline Ficus americana & Inga sapindoides & Mauria heterophylla & Piper arboreum \\
\hline Ficus citrifolia & Inga stenoptera & Maytenus durifolia & Piper umbellatum \\
\hline Ficus gomelleira & Inga striata & Melochia lupulina & Piptadenia flava \\
\hline Ficus insipida & Ipomoea carnea & Miconia laevigata & Piscidia carthagenensis \\
\hline Ficus jacobii & Ipomoea pauciflora & Mimosa acantholoba & Pisonia aculeata \\
\hline Ficus maxima & Ipomoea philomega & Mimosa myriadenia & Pithecellobium excelsum \\
\hline Ficus nymphaeifolia & Jacquemontia floribunda & Mimosa pectinatipinna & $\begin{array}{l}\text { Platymiscium } \\
\text { dimorphandrum }\end{array}$ \\
\hline Ficus obtusifolia & Jatropha clavuligera & Monteverdia retusa & $\begin{array}{l}\text { Pleuropetalum } \\
\text { pleiogynum }\end{array}$ \\
\hline Ficus pertusa & Jatropha curcas & Morus celtidifolia & Pleuropetalum sprucei \\
\hline Ficus sarmentosa & Jatropha humboldtiana & Mouriri myrtilloides & Poiretia punctata \\
\hline
\end{tabular}




\section{Continuación}

\begin{tabular}{|c|c|c|c|}
\hline \multicolumn{4}{|c|}{ Especies } \\
\hline Sapium glandulosum & Sorocea trophoides & Pouteria glomerata & Triplaris cumingiana \\
\hline Sapium laurifolium & $\begin{array}{l}\text { Sphinctanthus } \\
\text { aurantiacus }\end{array}$ & Pouzolzia occidentalis & Triplaris peruviana \\
\hline Schaefferia serrata & Spondias mombin & Pradosia montana & Triplaris weigeltiana \\
\hline Schrebera americana & $\begin{array}{l}\text { Stigmaphyllon } \\
\text { ellipticum }\end{array}$ & Pradosia mutisii & Triumfetta semitriloba \\
\hline Seguieria americana & Tabebuia chrysea & Prestonia mollis & Urera baccifera \\
\hline Senegalia polyphylla & Tabebuia impetiginosa & Prockia pentamera & Urera caracasana \\
\hline Senegalia riparia & Tabebuia ochracea & Prosopis juliflora & Vachellia aroma \\
\hline Senegalia tenuifolia & Tartagalia roseorum & Prosopis pallida & Vachellia macracantha \\
\hline $\begin{array}{l}\text { Senegalia } \\
\text { weberbaueri }\end{array}$ & Tecoma rosaefolia & Prunus subcorymbosa & Vallesia glabra \\
\hline Senna atomaria & Tecoma stans & $\begin{array}{l}\text { Pseudobaccharis } \\
\text { inamoena }\end{array}$ & Varronia macrocephala \\
\hline Senna galegifolia & $\begin{array}{l}\text { Tecoma } \\
\text { weberbaueriana }\end{array}$ & $\begin{array}{l}\text { Pseudobombax } \\
\text { cajamarcanus }\end{array}$ & Vasconcellea parviflora \\
\hline Senna macranthera & Terminalia valverdeae & Psidium guajava & $\begin{array}{l}\text { Verbesina lopez- } \\
\text { mirandae }\end{array}$ \\
\hline Senna mollissima & Tessaria integrifolia & Psidium rostratum & $\begin{array}{l}\text { Vernonanthura } \\
\text { phosphorica }\end{array}$ \\
\hline Senna ruiziana & $\begin{array}{l}\text { Tetrasida } \\
\text { chachapoyensis }\end{array}$ & Psidium rutidocarpum & Vernonia patens \\
\hline Senna spectabilis & Tetrasida serrulata & Psychotria horizontalis & Vitex gigantea \\
\hline Serjania ampelopsis & Tournefortia maculata & Pterocarpus amazonum & Wissadula excelsior \\
\hline $\begin{array}{l}\text { Sideroxylon } \\
\text { obtusifolium }\end{array}$ & Tournefortia ternifolia & Pterocarpus rohrii & Witheringia solanacea \\
\hline Simira ecuadorensis & Toxosiphon carinatus & Quararibea wittii & Ximenia americana \\
\hline Simira rubescens & $\begin{array}{l}\text { Toxosiphon } \\
\text { macropodus }\end{array}$ & Randia ferox & Xylosma benthamii \\
\hline Simira williamsii & Trema micrantha & Ricinus communis & Zamia poeppigiana \\
\hline Solanum caripense & Tricerma octogonum & Rinorea viridifolia & Zanthoxylum caribaeum \\
\hline Solanum hutchisonii & Trichilia elegans & Rudgea cornifolia & Zanthoxylum fagara \\
\hline Solanum leucocarpon & Trichilia hirta & Ruprechtia aperta & $\begin{array}{l}\text { Zanthoxylum } \\
\text { martinicense }\end{array}$ \\
\hline $\begin{array}{l}\text { Solanum } \\
\text { monadelphum }\end{array}$ & Trichilia moschata & Ruprechtia tenuiflora & Zanthoxylum rigidum \\
\hline $\begin{array}{l}\text { Solanum } \\
\text { pachyandrum }\end{array}$ & Trichilia pallida & Salix humboldtiana & Zanthoxylum sprucei \\
\hline Solanum umbellatum & Trichilia rubra & Salvia consobrina & Zapoteca caracasana \\
\hline Sorocea sprucei & Trichilia surinamensis & Sapindus saponaria & Ziziphus thyrsiflora \\
\hline
\end{tabular}


Anexo 5. Lista de especies de los bosques secos de Tumbes-Piura en estado de conservación y/o amenaza

\begin{tabular}{|c|c|c|c|c|c|c|c|c|}
\hline \multicolumn{5}{|c|}{ Especies } & \multicolumn{4}{|c|}{ Estado de conservación y/o amenaza } \\
\hline ID & Familia & $\begin{array}{c}\text { Nombre } \\
\text { científico citado }\end{array}$ & $\begin{array}{l}\text { Nombre } \\
\text { científico } \\
\text { aceptado }\end{array}$ & $\begin{array}{l}\text { Nombre } \\
\text { común }\end{array}$ & IUCN (2018) & $\begin{array}{c}\text { Apéndices CITES } \\
\text { (2016) }\end{array}$ & MINAGRI (2006) & MINAGRI (2016) \\
\hline 1 & Fabaceae & Acacia huarango & Vachellia aroma & Aromo & Ninguna & Ninguna & Casi amenazado (NT) & Ninguna \\
\hline 2 & Fabaceae & $\begin{array}{l}\text { Acacia } \\
\text { macracantha }\end{array}$ & $\begin{array}{l}\text { Vachellia } \\
\text { macracantha }\end{array}$ & Faique & Ninguna & Ninguna & Casi amenazado (NT) & Vulnerable (VU) \\
\hline 3 & Betulaceae & Alnus acuminata & $\begin{array}{l}\text { Alnus } \\
\text { acuminata }\end{array}$ & Aliso & $\begin{array}{l}\text { Preocupación } \\
\text { menor }\end{array}$ & Ninguna & Vulnerable (VU) & Casi amenazado (NT) \\
\hline 4 & Burseraceae & $\begin{array}{l}\text { Bursera } \\
\text { graveolens }\end{array}$ & $\begin{array}{l}\text { Bursera } \\
\text { graveolens }\end{array}$ & Palo santo & Ninguna & Ninguna & Peligro crítico (CR) & Peligro crítico (CR) \\
\hline 5 & Fabaceae & $\begin{array}{l}\text { Caesalpinia } \\
\text { paipai }\end{array}$ & $\begin{array}{l}\text { Caesalpinia } \\
\text { paipai }\end{array}$ & Charán & $\begin{array}{l}\text { Preocupación } \\
\text { menor }\end{array}$ & Ninguna & Ninguna & Ninguna \\
\hline 6 & Fabaceae & $\begin{array}{l}\text { Caesalpinia } \\
\text { spinosa }\end{array}$ & $\begin{array}{l}\text { Caesalpinia } \\
\text { spinosa }\end{array}$ & Tara & Ninguna & Ninguna & Vulnerable (VU) & Ninguna \\
\hline 7 & Capparaceae & $\begin{array}{l}\text { Capparis } \\
\text { avicenniifolia }\end{array}$ & $\begin{array}{l}\text { Beautempsia } \\
\text { avicenniifolia }\end{array}$ & Vichayo & Ninguna & Ninguna & Ninguna & Vulnerable (VU) \\
\hline 8 & Capparaceae & $\begin{array}{l}\text { Capparis } \\
\text { ovalifolia }\end{array}$ & $\begin{array}{l}\text { Beautempsia } \\
\text { avicenniifolia }\end{array}$ & Vichayo & Ninguna & Ninguna & Ninguna & Ninguna \\
\hline 9 & Malvaceae & $\begin{array}{l}\text { Ceiba } \\
\text { trischistandra }\end{array}$ & $\begin{array}{l}\text { Ceiba } \\
\text { trischistandra }\end{array}$ & Ceibo & Ninguna & Ninguna & Ninguna & Ninguna \\
\hline 10 & Cannabaceae & Celtis triflora & Celtis iguanaea & Palo blanco & Ninguna & Ninguna & Peligro crítico (CR) & Ninguna \\
\hline
\end{tabular}




\begin{tabular}{|c|c|c|c|c|c|c|c|c|}
\hline \multicolumn{5}{|c|}{ Especies } & \multicolumn{4}{|c|}{ Estado de conservación y/o amenaza } \\
\hline ID & Familia & $\begin{array}{c}\text { Nombre } \\
\text { científico citado }\end{array}$ & $\begin{array}{c}\text { Nombre científico } \\
\text { aceptado }\end{array}$ & Nombre común & IUCN (2018) & $\begin{array}{c}\text { Apéndices CITES } \\
\text { (2016) }\end{array}$ & MINAGRI (2006) & MINAGRI (2016) \\
\hline 11 & Fabaceae & $\begin{array}{l}\text { Cercidium } \\
\text { praecox }\end{array}$ & Parkinsonia praecox & Palo verde & Ninguna & Ninguna & Ninguna & Ninguna \\
\hline 12 & Bixaceae & \begin{tabular}{|l} 
Cochlospermum \\
vitifolium
\end{tabular} & Cochlospermum vitifolium & Polo polo & Ninguna & Ninguna & En peligro (EN) & Ninguna \\
\hline 13 & Capparaceae & $\begin{array}{l}\text { Colicodendron } \\
\text { scabridum }\end{array}$ & Colicodendron scabridum & Sapote & Ninguna & Ninguna & Peligro crítico (CR) & En peligro (EN) \\
\hline 14 & Boraginaceae & Cordia lutea & Cordia lutea & Overo & Ninguna & Ninguna & Ninguna & Ninguna \\
\hline 15 & Malvaceae & Eriotheca ruizii & Eriotheca ruizii & Pasallo & Ninguna & Ninguna & Ninguna & Ninguna \\
\hline 16 & Fabaceae & $\begin{array}{l}\text { Erythrina } \\
\text { smithiana }\end{array}$ & Erythrina smithiana & Porotillo, huayrul & Ninguna & Ninguna & Ninguna & Ninguna \\
\hline 17 & Moraceae & \begin{tabular}{|l} 
Ficus \\
nymphaeifolia
\end{tabular} & Ficus nymphaeifolia & Higuerón & Ninguna & Ninguna & Ninguna & Ninguna \\
\hline 18 & Fabaceae & Geoffroea striata & Geoffroea spinosa & Almendro de pepa & $\begin{array}{l}\text { Preocupación } \\
\text { menor }\end{array}$ & Ninguna & Ninguna & Ninguna \\
\hline 19 & Solanaceae & $\begin{array}{l}\text { Grabowskia } \\
\text { boerhaaviifolia }\end{array}$ & $\begin{array}{l}\text { Grabowskia } \\
\text { boerhaaviifolia }\end{array}$ & Canutillo & Ninguna & Ninguna & Ninguna & Vulnerable (VU) \\
\hline 20 & Fabaceae & $\begin{array}{l}\text { Leucaena } \\
\text { trichodes }\end{array}$ & Leucaena trichodes & Shapa & Ninguna & Ninguna & Ninguna & Ninguna \\
\hline
\end{tabular}




\begin{tabular}{|c|c|c|c|c|c|c|c|c|}
\hline \multicolumn{5}{|c|}{ Especies } & \multicolumn{4}{|c|}{ Estado de conservación y/o amenaza } \\
\hline ID & Familia & $\begin{array}{c}\text { Nombre científico } \\
\text { citado }\end{array}$ & $\begin{array}{c}\text { Nombre científico } \\
\text { aceptado }\end{array}$ & $\begin{array}{l}\text { Nombre } \\
\text { común }\end{array}$ & IUCN (2018) & $\begin{array}{l}\text { Apéndices } \\
\text { CITES (2016) }\end{array}$ & MINAGRI (2006) & MINAGRI (2016) \\
\hline 21 & Anacardiaceae & \begin{tabular}{|l|}
$\begin{array}{l}\text { Loxopterygium } \\
\text { huasango }\end{array}$ \\
\end{tabular} & Loxopterygium huasango & Hualtaco & Ninguna & Ninguna & Peligro crítico (CR) & Peligro crítico (CR) \\
\hline 22 & Muntingiaceae & $\begin{array}{l}\text { Muntingia } \\
\text { calabura }\end{array}$ & Muntingia calabura & Cerezo & Ninguna & Ninguna & Ninguna & Ninguna \\
\hline 23 & Fabaceae & $\begin{array}{l}\text { Parkinsonia } \\
\text { aculeata }\end{array}$ & Parkinsonia aculeata & $\begin{array}{l}\text { Palo verde, } \\
\text { azote de } \\
\text { cristo }\end{array}$ & Ninguna & Ninguna & Ninguna & Ninguna \\
\hline 24 & Fabaceae & $\begin{array}{l}\text { Parkinsonia } \\
\text { praecox }\end{array}$ & Parkinsonia praecox & Palo verde & Ninguna & Ninguna & Ninguna & Ninguna \\
\hline 25 & Fabaceae & \begin{tabular}{|l|}
$\begin{array}{l}\text { Pithecellobium } \\
\text { excelsum }\end{array}$ \\
\end{tabular} & Pithecellobium excelsum & $\begin{array}{l}\text { Chaquiro, } \\
\text { Chaquirón } \\
\end{array}$ & Ninguna & Ninguna & Ninguna & Ninguna \\
\hline 26 & Fabaceae & \begin{tabular}{|l|} 
Pithecellobium \\
multiflorum
\end{tabular} & Albizia multiflora & Angolo & Ninguna & Ninguna & Ninguna & Ninguna \\
\hline 27 & Fabaceae & Prosopis limensis & Prosopis pallida & Algarrobo & Ninguna & Ninguna & Vulnerable (VU) & Peligro crítico (CR) \\
\hline 28 & Fabaceae & Prosopis pallida & Prosopis pallida & Algarrobo & Ninguna & Ninguna & Vulnerable (VU) & En peligro (EN) \\
\hline 29 & Bignoniaceae & $\begin{array}{l}\text { Tabebuia } \\
\text { billbergii }\end{array}$ & Handroanthus billbergii & Guayacán & Ninguna & Ninguna & Ninguna & Ninguna \\
\hline 30 & Bignoniaceae & $\begin{array}{l}\text { Tabebuia } \\
\text { chrysantha }\end{array}$ & \begin{tabular}{|l} 
Handroanthus \\
chrysanthus
\end{tabular} & Madero negro & Ninguna & Ninguna & Ninguna & Ninguna \\
\hline 31 & Apocynaceae & Vallesia glabra & Vallesia glabra & Cun cun & \begin{tabular}{|l|} 
Preocupación \\
menor
\end{tabular} & Ninguna & Ninguna & Ninguna \\
\hline
\end{tabular}


Anexo 6. Lista de expertos locales en los bosques secos de Tumbes-Piura

\begin{tabular}{|c|c|c|c|c|c|}
\hline Departamento & $\begin{array}{l}\text { Nombres y } \\
\text { apellidos }\end{array}$ & Institución & Cargo & Correo electrónico & Teléfono móvil \\
\hline \multirow{4}{*}{ Lambayeque } & $\begin{array}{l}\text { Carlos Calderón } \\
\text { Vargas }\end{array}$ & $\begin{array}{l}\text { Proyecto Algarrobo- } \\
\text { INRENA }\end{array}$ & Ex Coordinador Lambayeque & forestalacv@gmail.com & 990321012 \\
\hline & $\begin{array}{l}\text { Romelio Díaz } \\
\text { Fuentes }\end{array}$ & $\begin{array}{l}\text { Instituto Nacional de } \\
\text { Innovación Agraria } \\
\text { (INIA) } \\
\end{array}$ & Coordinador EEA-Vista Florida & rdiazf@inia.gob.pe & 960954787 \\
\hline & $\begin{array}{l}\text { Patricia Medina } \\
\text { Llerena }\end{array}$ & Pronaturaleza & Ex-Coordinadora de campo del proyecto & pmedinall@yahoo.es & 945388267 \\
\hline & Juan Alarcón Díaz & ATFFS Lambayeque & Especialista forestal & jalarcon@serfor.gob.pe & 998738631 \\
\hline \multirow{4}{*}{ Piura } & $\begin{array}{l}\text { Luis Albán } \\
\text { Contreras }\end{array}$ & SUNASS & Ex-Coordinador de proyecto FOSEFOR & lalbanc@gmail.com & 969680560 \\
\hline & $\begin{array}{l}\text { Cristhian } \\
\text { Saldarriaga } \\
\text { Sánchez } \\
\end{array}$ & $\begin{array}{l}\text { Gobierno Regional de } \\
\text { Piura }\end{array}$ & Especialista forestal & foresdry@gmail.com & 970003306 \\
\hline & $\begin{array}{l}\text { César Talledo } \\
\text { Mendoza }\end{array}$ & $\begin{array}{l}\text { Gobierno Regional de } \\
\text { Piura }\end{array}$ & Coordinador de Proyecto NORBOSQUE & ctalledo@regionpiura.gob.pe & 969999358 \\
\hline & Juan Otivo Meza & ATFFS Piura & Administrador técnico & jotivo@serfor.gob.pe & 939414712 \\
\hline \multirow{3}{*}{ Tumbes } & $\begin{array}{l}\text { Miguel Puescas } \\
\text { Chully }\end{array}$ & $\begin{array}{l}\text { Universidad Nacional } \\
\text { de Tumbes }\end{array}$ & $\begin{array}{l}\text { Docente UNT, Coordinador programa } \\
\text { doctorado ciencias ambientales-EPG }\end{array}$ & mpuescasc@untumbes.edu.pe & 984098871 \\
\hline & $\begin{array}{l}\text { Juan La Rosa } \\
\text { Boggio }\end{array}$ & SERNANP & $\begin{array}{l}\text { Jefe de Parque Nacional Cerros de Amotape y } \\
\text { Reserva Nacional de Tumbes }\end{array}$ & jlarosa@sernanp.gob.pe & 972918733 \\
\hline & $\begin{array}{l}\text { Wilmer Rojas } \\
\text { Agurto }\end{array}$ & $\begin{array}{l}\text { Gobierno Regional de } \\
\text { Tumbes }\end{array}$ & Especialista forestal & wrojas04@hotmail.com & 972896100 \\
\hline
\end{tabular}


Anexo 7. Lista de especies de interés de los bosques secos del Marañon

\begin{tabular}{|c|c|c|c|c|c|c|c|c|c|}
\hline \multicolumn{3}{|c|}{ Especie } & \multicolumn{3}{|c|}{ Estado de conservación y/o amenaza } & \multicolumn{3}{|c|}{ Endemismo } & \multirow{2}{*}{$\begin{array}{c}\begin{array}{c}\text { Usos en prácticas } \\
\text { agroforestales }\end{array} \\
\begin{array}{c}\text { Marcelo Peña et al. } \\
\text { (2010) }\end{array}\end{array}$} \\
\hline ID & Familia & Nombre científico & IUCN (2018) & MINAGRI (2016) & $\begin{array}{l}\text { Marcelo Peña } \\
\text { et al. (2010) }\end{array}$ & $\begin{array}{l}\text { León et al. } \\
\text { (2006) }\end{array}$ & $\begin{array}{c}\text { Marcelo } \\
\text { Peña et al. } \\
\text { (2010) }\end{array}$ & $\begin{array}{l}\text { Marcelo } \\
\text { Peña et } \\
\text { al. (2015) }\end{array}$ & \\
\hline 1 & Malvaceae & Abutilon pedunculare & Ninguna & Ninguna & En peligro & $\mathrm{Si}$ & No & $\mathrm{Si}$ & Sin información \\
\hline 2 & Fabaceae & Acacia aroma & Ninguna & Ninguna & Ninguna & No & No & No & $\begin{array}{l}\text { Sistemas silvopastoriles, } \\
\text { sombra }\end{array}$ \\
\hline 3 & Fabaceae & Albizia multiflora & Ninguna & Ninguna & Ninguna & $\mathrm{Si}$ & No & No & $\begin{array}{l}\text { Sistemas silvopastoriles, } \\
\text { sombra }\end{array}$ \\
\hline 4 & Fabaceae & Albizia niopoides & Ninguna & Ninguna & Ninguna & No & No & No & Sombra \\
\hline 5 & Apocynaceae & Allamanda weberbaueri & Ninguna & Ninguna & Ninguna & $\mathrm{Si}$ & No & $\mathrm{Si}$ & Sin información \\
\hline 6 & Fabaceae & Anadenanthera colubrina & Ninguna & Ninguna & Ninguna & No & No & No & $\begin{array}{l}\text { Sistemas agroforestales } \\
\text { y silvopastoriles }\end{array}$ \\
\hline 7 & Aristolochiaceae & $\begin{array}{l}\text { Aristolochia } \\
\text { chachapoyensis }\end{array}$ & Ninguna & Ninguna & Ninguna & Si & No & No & Sin información \\
\hline 8 & Aristolochiaceae & Aristolochia xerophytica & Ninguna & Ninguna & Ninguna & $\mathrm{Si}$ & No & No & Sin información \\
\hline 9 & Apocynaceae & Aspidosperma polyneuron & En peligro (EN) & Ninguna & Ninguna & No & No & No & $\begin{array}{l}\text { Sistemas silvopastoriles, } \\
\text { sombra }\end{array}$ \\
\hline 10 & Fabaceae & Bauhinia augusti & Vulnerable (VU) & Ninguna & En peligro & No & $\mathrm{Si}$ & $\mathrm{Si}$ & Sin información \\
\hline 11 & Boraginaceae & Cordia iguaguana & Ninguna & Ninguna & Vulnerable & $\mathrm{Si}$ & $\mathrm{Si}$ & $\mathrm{Si}$ & $\begin{array}{l}\text { Sistemas silvopastoriles, } \\
\text { sombra }\end{array}$ \\
\hline 12 & Boraginaceae & Cordia varronifolia & Ninguna & Ninguna & En peligro & Si & $\mathrm{Si}$ & $\mathrm{Si}$ & Sin información \\
\hline 13 & Fabaceae & Coursetia maraniona & Ninguna & Ninguna & Vulnerable & $\mathrm{Si}$ & $\mathrm{Si}$ & $\mathrm{Si}$ & Sin información \\
\hline 14 & Euphorbiaceae & Croton adipatus & Ninguna & Ninguna & Ninguna & $\mathrm{Si}$ & $\mathrm{Si}$ & $\mathrm{Si}$ & Sin información \\
\hline
\end{tabular}




\begin{tabular}{|c|c|c|c|c|c|c|c|c|c|}
\hline \multicolumn{3}{|r|}{ Especie } & \multicolumn{3}{|c|}{ Estado de conservación y/o amenaza } & \multicolumn{3}{|c|}{ Endemismo } & \multirow{2}{*}{$\begin{array}{c}\begin{array}{c}\text { Usos en prácticas } \\
\text { agroforestales }\end{array} \\
\text { Marcelo Peña et al. (2010) }\end{array}$} \\
\hline ID & Familia & Nombre científico & IUCN (2018) & $\begin{array}{l}\text { MINAGRI } \\
(2016)\end{array}$ & $\begin{array}{c}\text { Marcelo Peña et } \\
\text { al. (2010) }\end{array}$ & $\begin{array}{l}\text { León et } \\
\text { al. (2006) }\end{array}$ & $\begin{array}{c}\text { Marcelo } \\
\text { Peña et al. } \\
\text { (2010) }\end{array}$ & $\begin{array}{l}\text { Marcelo } \\
\text { Peña et al. } \\
\text { (2015) }\end{array}$ & \\
\hline 15 & Euphorbiaceae & Croton rubiginosus & Ninguna & Ninguna & Ninguna & $\mathrm{Si}$ & No & $\mathrm{Si}$ & Sin información \\
\hline 16 & Fabaceae & Cyathostegia matthewsii & Ninguna & Ninguna & Ninguna & No & No & No & Cercos vivos \\
\hline 17 & Rutaceae & Esenbeckia cornuta & Ninguna & Ninguna & Peligro crítico & $\mathrm{Si}$ & $\mathrm{Si}$ & $\mathrm{Si}$ & Sin información \\
\hline 18 & Fabaceae & Geoffroea spinosa & $\begin{array}{l}\text { Preocupación Menor } \\
\text { (LC) }\end{array}$ & Ninguna & Ninguna & No & No & No & Sombra, defensa ribereña \\
\hline 19 & Malvaceae & Guazuma ulmifolia & Ninguna & Ninguna & Ninguna & No & No & No & $\begin{array}{l}\text { Sistemas silvopastoriles, } \\
\text { sombra }\end{array}$ \\
\hline 20 & Euphorbiaceae & Jatropha humboldtiana & Ninguna & Ninguna & Vulnerable & $\mathrm{Si}$ & $\mathrm{Si}$ & $\mathrm{Si}$ & Sin información \\
\hline 21 & Fabaceae & Leucaena trichodes & Ninguna & Ninguna & Ninguna & No & No & No & Sombra de café \\
\hline 22 & Fabaceae & Maraniona lavinii & Ninguna & Ninguna & Peligro crítico & $\mathrm{Si}$ & $\mathrm{Si}$ & $\mathrm{Si}$ & Sin información \\
\hline 23 & Fabaceae & Mimosa incarum & Ninguna & Ninguna & En peligro & $\mathrm{Si}$ & $\mathrm{Si}$ & $\mathrm{Si}$ & $\begin{array}{l}\text { Setos vivos, fijación de } \\
\text { taludes }\end{array}$ \\
\hline 24 & Fabaceae & Mimosa pectinatipinna & Ninguna & Ninguna & $\begin{array}{l}\text { Preocupación } \\
\text { menor }\end{array}$ & $\mathrm{Si}$ & $\mathrm{Si}$ & $\mathrm{Si}$ & Estabilización de taludes \\
\hline 25 & Muntingiaceae & Muntingia calabura & Ninguna & Ninguna & Ninguna & No & No & No & Fijación de taludes \\
\hline 26 & Fabaceae & Parkinsonia peruviana & Peligro Crítico (CR) & Ninguna & Ninguna & $\mathrm{Si}$ & No & $\mathrm{Si}$ & Sin información \\
\hline 27 & Fabaceae & Piptadenia weberbaueri & Vulnerable (VU) & Ninguna & Ninguna & $\mathrm{Si}$ & No & $\mathrm{Si}$ & Sin información \\
\hline 28 & Fabaceae & Prosopis juliflora & Ninguna & $\begin{array}{l}\text { En Peligro } \\
\text { (EN) }\end{array}$ & Ninguna & No & No & No & $\begin{array}{l}\text { Sistemas silvopastoriles, } \\
\text { sombra }\end{array}$ \\
\hline
\end{tabular}




\begin{tabular}{|c|c|c|c|c|c|c|c|c|c|}
\hline \multirow[b]{2}{*}{ ID } & \multicolumn{2}{|r|}{ Especie } & \multicolumn{3}{|c|}{ Estado de conservación y/o amenaza } & \multicolumn{3}{|c|}{ Endemismo } & \multirow{2}{*}{$\begin{array}{l}\text { Usos en prácticas agroforestales } \\
\text { Marcelo Peña et al. (2010) }\end{array}$} \\
\hline & Familia & Nombre científico & $\begin{array}{l}\text { IUCN } \\
(2018)\end{array}$ & $\begin{array}{l}\text { MINAGRI } \\
(2016)\end{array}$ & $\begin{array}{c}\text { Marcelo } \\
\text { Peña et al. } \\
(2010)\end{array}$ & $\begin{array}{l}\text { León et al. } \\
\text { (2006) }\end{array}$ & $\begin{array}{c}\text { Marcelo } \\
\text { Peña et al. } \\
\text { (2010) }\end{array}$ & $\begin{array}{l}\text { Marcelo } \\
\text { Peña et al. } \\
\text { (2015) }\end{array}$ & \\
\hline 29 & Malvaceae & Pseudobombax cajamarcanus & Ninguna & Ninguna & $\begin{array}{l}\text { Peligro } \\
\text { crítico }\end{array}$ & No & $\mathrm{Si}$ & Si & Sin información \\
\hline 30 & Polygonaceae & Ruprechtia aperta & Ninguna & Ninguna & Vulnerable & $\mathrm{Si}$ & $\mathrm{Si}$ & $\mathrm{Si}$ & Sin información \\
\hline 31 & Sapindaceae & Sapindus saponaria & Ninguna & Ninguna & Ninguna & No & No & No & $\begin{array}{l}\text { Sistemas silvopastoriles, sombra, } \\
\text { cercos }\end{array}$ \\
\hline 32 & Fabaceae & Senegalia polyphylla & Ninguna & Ninguna & Ninguna & No & No & No & Sombra \\
\hline 33 & Fabaceae & Senna mollissima & Ninguna & Ninguna & Ninguna & No & No & No & Sombra \\
\hline 34 & Sapotaceae & Sideroxylon obtusifolium & Ninguna & Ninguna & Ninguna & No & No & No & Sistemas silvopastoriles, sombra \\
\hline 35 & Bignoniaceae & Tecoma rosifolia & Ninguna & Ninguna & Vulnerable & No & $\mathrm{Si}$ & $\mathrm{Si}$ & Estabilización de taludes \\
\hline 36 & Asteraceae & Tessaria integrifolia & Ninguna & Ninguna & Ninguna & No & No & No & Defensa ribereña \\
\hline 37 & Malvaceae & Tetrasida chachapoyensis & Ninguna & Ninguna & Vulnerable & $\mathrm{Si}$ & $\mathrm{Si}$ & $\mathrm{Si}$ & Sin información \\
\hline 38 & Malvaceae & Tetrasida serrulata & Ninguna & Ninguna & En peligro & $\mathrm{Si}$ & $\mathrm{Si}$ & $\mathrm{Si}$ & Sin información \\
\hline 39 & Fabaceae & Vachellia macracantha & Ninguna & $\begin{array}{l}\text { Vulnerable } \\
\text { (VU) }\end{array}$ & Ninguna & No & No & No & Sistemas silvopastoriles, sombra \\
\hline
\end{tabular}




\section{Referencias}

Albán, L. 2005. Fortalecimiento de las capacidades locales para la producción de semillas de seis especies forestales de los bosques secos de la región Piura: Informe final técnico y financiero. Centro Ideas. Programa Andino de Fomento de Semillas Forestales. Piura, Perú. 61 p.

Angulo, F. 2009. Los bosques secos del noroeste del Perú: una invitación a la reflexión. Lima, Perú. 5 p.

Cabrejos, C., J. Namoc y E. Cunaique. 2006. Estudio fenológico de especies forestales nativas de Bosque Seco-Alto Piura. Centro Ideas. Programa Andino de Fomento de Semillas Forestales. Piura, Peru. 187 p.

Castro, A. 2007. Estudio de las propiedades de la fibra vegetal "kapok" de Ceiba trischistandra en función a la clase diamétrica y nivel altitudinal, proveniente del Bosque Seco de Bolívar, Cajamarca. Tesis para optar el Título Profesional de Ingeniero Forestal. Universidad Nacional Agraria La Molina. Lima, Perú. 146p.

Cuellar, J., Ugarte, J. Vilcapoma, E. 2016. Las semillas forestales en el Perú: Desafíos y Oportunidades. Instituto Nacional de Innovación Agraria (INIA). Lima, Perú. 76 p IUCN (International Union of Conservation of Nature). 2018. The IUCN Red List of Threatened Species. Disponible en: http://www.iucnredlist.org/.

Lazo, J. 2014. Estrategias para la restauración de ámbitos degradados en el Santuario Histórico Bosque de Pómac. SERNANP. Lambayeque, Perú. 64 p.

León, B., J. Roque, C. Ulloa, N. Pitman, P.M. Jorgensen y A. Cano (Eds). 2006. El libro rojo de las Plantas endémicas del Perú. Rev.Perú.biol. Número especial 13(2): 1-967 Linares-Palomino, R. 2004. Los Bosques Tropicales Estacionalmente Secos: El concepto de los bosques secos en el Perú. Arnaldoa 11(1):85-102

Llontop, J. 2013. Impactos del cambio climático y medidas de adaptación para la conservación del Bosque Seco y sostenibilidad de la cadena de valor de derivados del algarrobo en la región Piura. Gobierno Regional de Piura, GIZ.

Marcelo-Peña, J.L. 2008. Vegetación leñosa, endemismos y estado de conservación en los bosques estacionalmente secos de Jaén, Perú. Rev. Perú Biol. 15 (1):43-52 
Marcelo-Peña, J.L., R.T. Pennington, C. Reynel y P. Zevallos. 2010. Guía ilustrada de la flora leñosa de los Bosques Estacionalmente Secos de Jaén, Perú. Universidad Nacional Agraria La Molina y Royal Botanic Garden Edinburgh. 290p.

Marcelo-Peña, J.L., I. Huamantupa, T. Särkinen y M. Tomazello. 2015. Identying conservation priority areas in the Marañon Valley (Peru) based on Floristic inventories. Edingburgh Journal of Botany 1-29

MINAGRI (Ministerio de Agricultura y Riego). 2006. Reglamento de Semillas Forestales (Decreto Supremo N042-2006-AG). Lima, Perú. 12 p.

MINAGRI (Ministerio de Agricultura y Riego). 2006. Decreto Supremo que aprueba la Categorización de Especies Amenazadas de Flora Silvestre (D.S. Nº43-2006-AG). Lima, Perú. 13 p.

MINAGRI (Ministerio de Agricultura y Riego). 2014. Decreto Supremo que aprueba la Actualización y Categorización de las Especies Amenazadas de Fauna Silvestre Legalmente Protegidas (D.S.004-2014-MINAGRI). Lima, Perú. 68p.

MINAGRI (Ministerio de Agricultura y Riego). 2016. Publicación preliminar de los Anexo I y II que contienen la Clasificación Oficial de Especies de Flora Silvestre categorizadas como amenazadas (Resolución Ministerial 0505-2016-MINAGRI). Lima, Perú. 18pMINAM (Ministerio del Ambiente). 2011. El Perú de los bosques. Lima, Perú, $73 \mathrm{p}$.

MINAM (Ministerio del Ambiente). 2011. El Perú de los bosques. Lima, Perú, 73 p. MINAM (Ministerio del Ambiente). 2011. Estudio para la identificación de áreas degradadas y propuesta de monitoreo. Dirección General de Ordenamiento Territorial Ambiental. Lima, Perú. $44 \mathrm{p}$

MINAM (Ministerio del Ambiente). 2016. Lista de especies de flora peruana incluida en los Apéndices I, II, III CITES. Dirección General de Diversidad Biológica Programa de Asistencia Técnica PAT-USAID/MINAM, Lima, Perú.155 p.

Odar, J. 2010. Especies forestales del Coto de Caza El Angolo, Sullana. Tesis para optar el Título Profesional de Biólogo. Universidad Nacional de Piura. Piura, Perú. 84 p. 
SENASA (Servicio Nacional de Sanidad Agraria). 2016. Situación fitosanitaria del algarrobo (Prosopis pallida) en la costa norte del Perú. Subdirección de Análisis de Riesgo y Vigilancia Fitosanitaria. MINAGRI. Lima, Perú. 47 p

Whaley, O., A. Orellana, I. Pérez, M. Tenorio, F. Quinteros, M. Mendoza y O. Pecho. 2010. Plantas y vegetación de Ica, Perú-Un recurso para su restauración y conservación. Royal Botanic Gardens, Kew. 98 p 


\section{Documentos de Trabajo de ICRAF (Working Paper Series)}

240. The national agroforestry policy of India: experiential learning in development and delivery phases. http://dx.doi.org/10.5716/WP16143.PDF

241. Agroforestry and forestry in Sulawesi series: Livelihood strategies and land-use system dynamics in Gorontalo. http://dx.doi.org/10.5716/WP16157.PDF

242. Seri Agroforestri dan Kehutanan di Sulawesi: Strategi mata pencaharian dan dinamika system penggunaan lahan di Gorontalo. http://dx.doi.org/10.5716/WP16158.PDF

243. Ruang, Gender dan Kualitas Hidup Manusia: Sebuah studi Gender pada komunitas perantau dan pengelola kebun di Jawa Barat. http://dx.doi.org/10.5716/WP16159.PDF

244. Gendered knowledge and perception in managing grassland areas in East Sumba, Indonesia. http://dx.doi.org/10.5716/WP16160.PDF

245. Pengetahuan dan persepsi masyarakat pengelola padang aavana, Sebuah Kajian Gender di Sumba Timur. http://dx.doi.org/10.5716/WP16161.PDF

246. Dinamika Pengambilan Keputusan pada komunitas perantau dan pengelola kebun di Jawa Barat. http://dx.doi.org/10.5716/WP16162.PDF

247. Gaharu (eaglewood) domestication: biotechnology, markets and agroforestry options. http://dx.doi.org/10.5716/WP16163.PDF

248. Marine habitats of the Lamu-Kiunga coast: an assessment of biodiversity value, threats and opportunities. http://dx.doi.org/10.5716/WP16167.PDF

249. Assessment of the biodiversity in terrestrial landscapes of the Witu protected area and surroundings, Lamu County, Kenya. http://dx.doi.org/10.5716/WP16172.PDF

250. An ecosystem services perspective on benefits that people derive from biodiversity of Coastal forests in Lamu County, Kenya http://dx.doi.org/10.5716/WP16173.PDF

251. Assessment of the biodiversity in terrestrial and marine landscapes of the proposed Laga Badana National Park and surrounding areas, Jubaland, Somalia. http://dx.doi.org/10.5716/WP16174.PDF 
252. Preferensi Petani terhadap Topik Penyuluhan dan Penyebaran Informasi Agroforestri di Indonesia http://dx.doi.org/10.5716/WP16181.PDF

253. Seri Agroforestri dan Kehutanan di Sulawesi: Keanekaragaman hayati jenis pohon pada hutan rakyat agroforestri di DAS Balangtieng, Sulawesi Selatan http://dx.doi.org/10.5716/WP16182.PDF

254. Potensi dan Tantangan dalam Pengembangan Skema Ko-Investasi Jasa Lingkungan di Kabupaten Buol, Indonesia. http://dx.doi.org/10.5716/WP17008.PDF

255. Keragaman Jenis Pohon dan Pemanfaatannya oleh Masyarakat di Kabupaten Buol, Indonesia. http://dx.doi.org/10.5716/WP17009.PDF

256. Kerentanan dan preferensi sistem pertanian petani di Kabupaten Buol, Indonesia http://dx.doi.org/10.5716/WP17010.PDF

257. Dinamika Perubahan Penggunaan/Tutupan Lahan Serta Cadangan Karbon di Kabupaten Buol, Indonesia. http://dx.doi.org/10.5716/WP17011.PDF

258. The effectiveness of the volunteer farmer trainer approach vis-à-vis other information sources in dissemination of livestock feed technologies in Uganda.

http://dx.doi.org/10.5716/WP17104.PDF

259. Agroforestry and forestry in Sulawesi series: Impact of agricultural-extension booklets on community livelihoods in South and Southeast Sulawesi.

http://dx.doi.org/10.5716/WP17125.PDF

260. Petani Menjadi Penyuluh, Mungkinkah? Sebuah Pendekatan Penyuluhan dari Petani ke Petani di Kabupaten Sumb Timur. http://dx.doi.org/10.5716/WP17145.PDF

261. Dampak Perubahan Tutupan Lahan terhadap Kondisi Hidrologi di Das Buol, Kabupaten Buol, Sulawesi Tengah: Simulasi dengan Model Genriver http://dx.doi.org/10.5716/WP17146.PDF

262. Analisis Tapak Mata Air Umbulan, Pasuruan, Jawa Timur. Kajian elemen biofisik dan persepsi masyarakat. http://dx.doi.org/10.5716/WP17147.PDF 
263. Planned comparisons demystified. http://dx.doi.org/10.5716/WP17354.PDF

264. Soil health decision support for NERC digital soil platforms: A survey report http://dx.doi.org/10.5716/WP17355.PDF

265. Seri Pembangunan Ekonomi Pedesaan Indonesia: Menanam di bukit gundul: Pengetahuan masyarakat lokal dalam upaya restorasi lahan di Sumba

Timur. http://dx.doi.org/10.5716/WP17356.PDF

266. Tree diversity and carbon stock in three districts of Kutai Timur, Pasir and Berau, East Kalimantan http://dx.doi.org/10.5716/WP17357.PDF

267. Tree Diversity and Carbon Stock in Various Land Use Systems of Banyuasin and Musi Banyuasin Districts, South Sumatera http://dx.doi.org/10.5716/WP17358.PDF

268. Tree diversity and carbon stock in various land cover systems of Jayapura, Jayawijaya and Merauke Districts, Papua Province http://dx.doi.org/10.5716/WP17359.PDF

269. Modelling tree production based on farmers' knowledge: case for kapok (Ceiba pentandra) and candlenut (Aleurites mollucana) under various agroforestry scenarios. http://dx.doi.org/10.5716/WP17361.PDF

270. The Impact of Land Cover and Climate Change on Present and Future Watershed Condition. Study case: Tugasan, Alanib and Kulasihan Sub-watershed of Manupali Watershed, Lantapan, Bukidnon, Philippines. http://dx.doi.org/10.5716/WP17362.PDF

271. Tree Diversity and Above-ground Carbon Stock estimation in Various Land use Systems in Banjarnegara, Banyumas and Purbalingga, Central Java.

http://dx.doi.org/10.5716/WP17363.PDF

272. Agroforestry and Forestry in Sulawesi series: Landscape Management Strategies in Sulawesi: Review of Intervention Options. http://dx.doi.org/10.5716/WP17364.PDF

273. Household Food-Security and Nutritional Status of Women and Children in Buol Regency, Central Sulawesi, Indonesia. http://dx.doi.org/10.5716/WP17365.PDF

274. Palm oil expansion in tropical forest margins or sustainability of production? Focal issues of regulations and private standards. http://dx.doi.org/10.5716/WP17366.PDF 
$\underline{2018}$

275. Decision analysis methods guide: Agricultural policy for nutrition http://dx.doi.org/10.5716/WP18001.PDF

276. Supporting human nutrition in Africa through the integration of new and orphan crops into food systems: Placing the work of the African Orphan Crops Consortium in context. http://dx.doi.org/10.5716/WP18003.PDF

277. Seri Pembangunan Ekonomi Pedesaan Indonesia. Pilihan Manajemen Budidaya Kacang Tanah sebagai Upaya untuk Memperbaiki Penghidupan Masyarakat Haharu.

http://dx.doi.org/10.5716/WP18004.PDF

278. Estudio de línea de base CCAFS a nivel de hogar en Nicaragua y Costa Rica

Fase de diagnóstico del estudio: "Contribución de la diversidad arbórea a los medios de vida para la adaptación y la mitigación al cambio climático

http://dx.doi.org/10.5716/WP18005.PDF

279. Understanding tree cover transition, drivers and stakeholder perspectives for effective landscape governance. A case study in Na Nhan commune, Dien Bien province, Vietnam. http://dx.doi.org/10.5716/WP18006.PDF

280. El Sistema "Quesungual": Agroforestería y manejo de suelos para la producción de maíz y frijol en laderas. http://dx.doi.org/10.5716/WP18007.PDF

281: Probabilistic Decision Modelling to Determine Impacts on Natural Resource Management and Livelihood Resilience in Marsabit County, Kenya.

http://dx.doi.org/10.5716/WP18008.PDF

282. Shifting discourse, shifting power: how is climate change mitigation and justice negotiated in Indonesia? http://dx.doi.org/10.5716/WP18009.PDF

283. Result of Land Use Planning and Land Administration (LULA) Implementation in South Sumatra, East Kalimantan, Central Java and Papua http://dx.doi.org/10.5716/WP18010.PDF

284. Farmers' preferences for training topics and dissemination of agroforestry information in Indonesia. http://dx.doi.org/10.5716/WP18015.PDF 
285. CSA-Diagnostic (CSA-Dx): A primer for investigating the 'climate-smartness' of ag technologies http://dx.doi.org/10.5716/WP18020.PDF

286. An analysis of the vulnerability of poor communities in Yunnan Province, China http://dx.doi.org/10.5716/WP18021.PDF

287. Gendered space and quality of life: gender study of out-migration and smallholding agroforestry communities in West Java Province, Indonesia.

http://dx.doi.org/10.5716/WP18024.PDF

288: Evaluation of UTZ certification coffee businesses in Guatemala, Honduras and Nicaragua. http://dx.doi.org/10.5716/WP18028.PDF

289. Agroforestry species of Peru: annotated list and contribution to prioritization for genetic conservation. http://dx.doi.org/10.5716/WP18029.PDF

290. Indonesia Rural Economic Development Series.Growing plants on a barren hill: local knowledge as part of land restoration in Sumba Timur, Indonesia.

http://dx.doi.org/10.5716/WP18030.PDF

291. Assessing the Downstream Socioeconomic Impacts of Agroforestry in Kenya http://dx.doi.org/10.5716/WP18033.PDF

\section{$\underline{2019}$}

292: Los árboles fuera del bosque en la NAMA forestal de Colombia. Elementos conceptuales para su contabilización. http://dx.doi.org/10.5716/WP19002.PDF

293: Gender and Adaptation: An Analysis of Poverty and Vulnerability in Yunnan, China. DOI: http://dx.doi.org/10.5716/WP19004.PDF

294: Tree Cover on Agricultural Land in the Asia Pacific Region DOI: http://dx.doi.org/10.5716/WP19005.PDF

295: What do we really know about the impacts of improved grain legumes and dryland cereals? A critical review of 18 impact studies DOI: http://dx.doi.org/10.5716/WP19006.PDF 
296: Breeders' views on the production of new and orphan crops in Africa: a survey of constraints and opportunities

DOI: $\underline{\text { http://dx.doi.org/10.5716/WP19007.PDF }}$

297: Biomass Resources in Rhino Camp and Imvepi Refugee Settlements and the Buffer Zone around these Settlements in West Nile, Uganda

DOI: http://dx.doi.org/10.5716/WP19031.PDF

298: Option for restocking woody biomass in refugee-hosting areas: Perspectives from communities in Rhino Camp and Imvepi Settlements, Uganda

DOI: http://dx.doi.org/10.5716/WP19032.PDF

299: Restoring ecosystems in refugee settlements using tree-based systems: The case of Rhino Camp and Imvepi Settlements in Uganda.

DOI: http://dx.doi.org/10.5716/WP19033.PDF

300: A theory-based evaluation of the Agroforestry Food Security Programme, Phase II in Malawi (AFSPII): Lessons for Scaling Up Complex Agronomic and Natural Resource Management Practices Developed and Tested in Research Settings.

DOI: http://dx.doi.org/10.5716/WP19036.PDF 
World Agroforestry (ICRAF) is a centre of scientific and development excellence that harnesses the benefits of trees for people and the environment. Leveraging the world's largest repository of agroforestry science and information, we develop knowledge practices, from farmers' fields to the global sphere, to ensure food security and environmental sustainability.

ICRAF is the only institution that does globally significant agroforestry research in and for all of the developing tropics. Knowledge produced by ICRAF enables governments, development agencies and farmers to utilize the power of trees to make farming and livelihoods more environmentally, socially and economically sustainable at multiple scales.

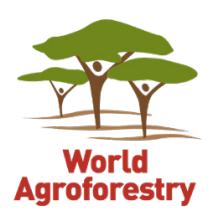

United Nations Avenue, Gigiri • PO Box 30677 • Nairobi, $00100 \cdot$ Kenya Telephone: +254207224000 or via USA +1 6508336645 Fax: +254207224001 or via USA +1 6508336646 Email: worldagroforestry@cgiar.org•www.worldagroforestry.org 